\title{
Acupoint Stimulation for Acne: A Systematic Review of Randomized Controlled Trials
}

\author{
Hui-juan Cao, PhD, * Guo-yan Yang, BSMed, ${ }^{*}$ Yu-yi Wang, BSMed, and Jian-ping Liu, PhD
}

\begin{abstract}
Background: Acupoint stimulation-including acupuncture, moxibustion, cupping, acupoint injection, and acupoint catgut embedding-has shown a beneficial effect for treating acne. However, comprehensive evaluation of current clinical evidence is lacking.

Objective: The aim of this review was to assess the effectiveness and safety of all acupoint stimulation techniques used to treat acne vulgaris.

Design: A systematic review was conducted. It included only randomized controlled trials on acupoint stimulation for acne. Six electronic databases were searched for English and Chinese language studies. All searches ended in May 2012. Studies were selected for eligibility and assessed for quality. RevMan 5.1 software was used for data analysis with an effect estimate presented as risk ratios $(R R)$ or mean difference (MD) with a 95\% confidence interval (CI). Patients: Studies with subjects who were diagnosed with acne vulgaris, or papulopustular, inflammatory, adolescent, or polymorphic acne—regardless of gender, age, and ethnicity—were included.

Intervention: Interventions included any acupoint stimulation technique-such as acupuncture, moxibustion, cupping, acupoint injection, and acupoint catgut embedding-compared with no treatment, placebo, or conventional pharmaceutical medication.

Main Outcome Measure: Reduction of signs and symptoms and presence of adverse effects were examined. Results: Forty-three trials involving 3453 patients with acne were included. The methodological quality of trials was generally poor in terms of randomization, blinding, and intention-to-treat analysis. Meta-analyses showed significant differences in increasing the number of cured patients between acupuncture plus herbal medicine and herbal medicine alone $(R R: 1.60 ; 95 \%$ CI: $1.19-2.14 ; P=0.002)$, and between acupuncture plus herbal facial mask and herbal facial mask alone $(R R: 2.14 ; 95 \%$ CI: $1.29-3.55 ; P=0.003)$. Cupping therapy was significantly better than pharmaceutical medications for increasing the number of cured patients $(R R: 2.11 ; 95 \%$ CI: 1.45-3.07; $P<0.0001)$. Serious adverse events were not reported in all included trials.
\end{abstract}

Conclusions: Acupoint stimulation therapies combined with other treatments appears to be effective for acne. However, further large, rigorously designed trials are needed to confirm these findings.

Key Words: Acne, Acupoint Stimulation, Systematic Review

\section{INTRODUCTION}

A CNE IS A CHRONIC INFLAMMATORY CONDITION of the skin. This condition most commonly affects areas where the sebaceous glands are largest and most abundant: the face; anterior trunk; and upper back. ${ }^{1}$ Mild acne is characterized by comedones, or blackheads, which are dilated pores with a plug of keratin. Moderate-to-severe acne is characterized by whiteheads (small cream-colored, domeshaped papules), red papules, pustules, or cysts. Scars, both 
those on the skin and emotional scars can last a lifetime. Acne affects $80 \%$ of adolescents (most commonly from 12 years of age), but it can also affect $54 \%$ of adult women and $40 \%$ of adult men (primarily those in their early or mid-20s). ${ }^{2}$

Acne treatment is aimed at decreasing inflammation of the lesions and accompanying discomfort with the ultimate goals of improving appearance and preventing or minimizing scarring and emotional distress. ${ }^{3}$ Effective prescription medications are available, although side-effects-such as local irritation, teratogenicity, dry skin, hyperlipidemia, and increased risk of depression-are reported. ${ }^{4,5}$ Overthe-counter preparations and herbal remedies, as well as skin- hygiene routines and dietary modifications may be recommended by dermatologists or, more often, are selfprescribed. ${ }^{4}$

In China, in addition to prescription medications, traditional Chinese therapies are used to treat acne. One such therapy is acupoint stimulation. Several methods can be applied: acupuncture with needles; moxibustion, which involves the controlled burning of material, typically mugwort (Artemisia vulgaris) herb, at certain points or areas of the body surface; cupping therapy, which involves applying suction by placing a vacuumized, usually by fire, cup or jar on acupoints or affected body surfaces to induce local hyperemia or hemostasis; acupoint injection, which involves injecting medication into an acupuncture point; and acupoint embedding, which involves embedding in the skin over the acupoint a small needle(s), or medicated catgut.

Acupoint-stimulation methods are based on the Traditional Chinese Medicine (TCM) view that acne is caused by any or a combination of several pathogenic factors. These include intense Lung Heat or Stomach Heat, Damp-Heat with Blood Stasis, and Qi (vital energy) Stagnation. As the condition becomes protracted, pathogenic Heat rises and accumulates in the skin and tissues, which brings on the lesions. ${ }^{6}$

Potential mechanisms of acupoint stimulation for acne are to relieve Heat toxicity, eliminate Dampness, regulate the Qi and Blood, and enhance immunologic function. ${ }^{6}$ Some studies also mention that acupuncture can stimulate and balance androgen levels to inhibit excess secretion of the sebaceous gland. ${ }^{7}$

Articles with clinical observations have reported the efficacy of acupoint-stimulation therapies for acne. A systematic review ${ }^{7}$ assessing seventeen TCM randomized controlled trials (RCTs) suggests that acupuncture and moxibustion are better than conventional pharmaceutical medication for reducing symptoms of acne. Another systematic review ${ }^{8}$ involving twenty-three trials of topical and oral complementary and alternative medicines (CAMs) concluded that, poor methodological quality aside, the evidence suggests that many of these therapies are biologically plausible. However, there has been no systematic review that evaluated the clinical evidence of all types of acupoint-stimulation therapies. This systematic review was performed to assess the effectiveness and safety of all acupoint-stimulation techniques used to treat acne vulgaris.

\section{METHODS}

\section{Inclusion Criteria}

Parallel-group RCTs were included in any data analysis with interventions for the treatment of acne vulgaris using any acupoint stimulation technique — such as acupuncture, moxibustion, cupping, acupoint injection, and acupoint catgut embedding - compared with no treatment, placebo, or conventional pharmaceutical medication. Comparisons also included a combination of acupoint-stimulation techniques, plus other therapies, versus the same other therapies alone. Participants who were diagnosed with acne vulgaris, or papulopustular, inflammatory, adolescent, or polymorphic acne-regardless of gender, age, and ethnicity-were included. Primary outcome measures were reduction of signs and symptoms and presence of adverse effects. Secondary outcome measures included post-treatment evaluation, participants' self-assessment of change in lesions after treatment, psychosocial outcomes, and quality of life (QoL) measurements.

\section{Identification and Selection of Studies}

Searches were conducted in the China Network Knowledge Infrastructure (CNKI, 1979-2012), Chinese Scientific Journals Database (VIP, 1989-2012), Wan Fang Database (1985-2012), Chinese Biomedicine (CBM, 1978-2012), Cochrane Central Register of Controlled Trials (CENTRAL, 1999-2012), and PubMed (1966-2012). All searches ended in May 2012. Search terms included acupuncture, electroacupuncture, auricular therapy, acupoint, meridian, combined with acne vulgaris and acne. Unpublished postgraduate theses in Chinese databases were also searched. No language restrictions were imposed. Three authors (H.-j.C., G.-y.Y., and Y.-y.W.) independently selected studies for eligibility and checked against the inclusion criteria.

\section{Data Extraction and Quality Assessment}

Three authors (H.-j.C., G.-y.Y., and Y.-y.W.) independently extracted population and intervention characteristics using self-designed data extraction templates. Disagreements were resolved by discussion with another author (J.-p.L).

Risk of bias for each study was conducted in accord with the Cochrane Handbook for Systematic Reviews of Intervention. ${ }^{9}$ Six criteria were applied, as follows: (1) selection bias (random-sequence generation and allocation 
concealment); (2) performance bias (blinding of participants and personnel); (3) detection bias (blinding of outcome assessment); (4) attrition bias (incomplete outcome data); (5) reporting bias (selective reporting); and (6) other bias. There were three potential bias judgments: (1) low risk; (2) high risk; and (3) unclear risk. A study was rated unclear risk when insufficient details were reported regarding what happened in the study. A judgment of unclear risk was also made when what happened in the study was known but the risk of bias was unknown or when an item was not relevant to the study, particularly for assessing blinding and incomplete outcome data or when the outcome assessed by the item had not been measured in the study.

\section{Data Analysis}

Dichotomous data were expressed as risk ratios $(R R)$ with a $95 \%$ confidence interval (CI). Continuous data were expressed as mean differences (MDs) with 95\% CIs. Statistical heterogeneity was tested by the $I^{2}$ test. RevMan 5.1 software (Cochrane Collaboration) was used for data analyses. Meta-analysis was used if the trials had acceptable homogeneity $\left(I^{2}<85 \%\right)$ of study design, participants, interventions, controls, and outcome measures. Meta-analyses were performed using fixed-effect ${ }^{9}$ models $\left(I^{2}<25 \%\right)$ for homogeneous studies and using random-effects methods prior to fixed-effect models when there was substantial heterogeneity $\left(25 \%<I^{2}<85 \%\right)$.

\section{RESULTS}

\section{Description of Studies}

After primary searches of six databases, 535 citations were identified. We excluded 484 studies because they did not meet inclusion criteria. Full-text articles for 51 studies were retrieved. Finally, 43 trials $^{10-52}$ were included in this review (Fig. 1). Characteristics of included trials are listed in Table 1. Among the included trials, fourteen studies ${ }^{11-15,19,21,26,31,35,42,43,45,46}$ were unpublished master's theses.

The forty-three trials involved a total of 3453 patients, with an average of 40 participants in each group. Age ranged between 13 and 43, and duration of disease varied from 1 week to 17 years. Ten trials $22,23,25,27,29,38,39,47,48,50$ did not report diagnostic criteria, two trials ${ }^{10,30}$ used international diagnostic criteria, and thirty-three trials reported using one of four TCM diagnostic criteria. ${ }^{53-56}$ Of the thirty-three trials, six trials $24,36,40,41,43,52$ did not report sources for diagnostic criteria, and eight trials ${ }^{11,15,17,20,31,34,37,51}$ used selfestablished diagnostic criteria for acne.

Interventions included acupuncture (electroacupuncture, auricular acupuncture, and ear point pressure), cupping therapy, acupoint injection, acupoint catgut embedding,

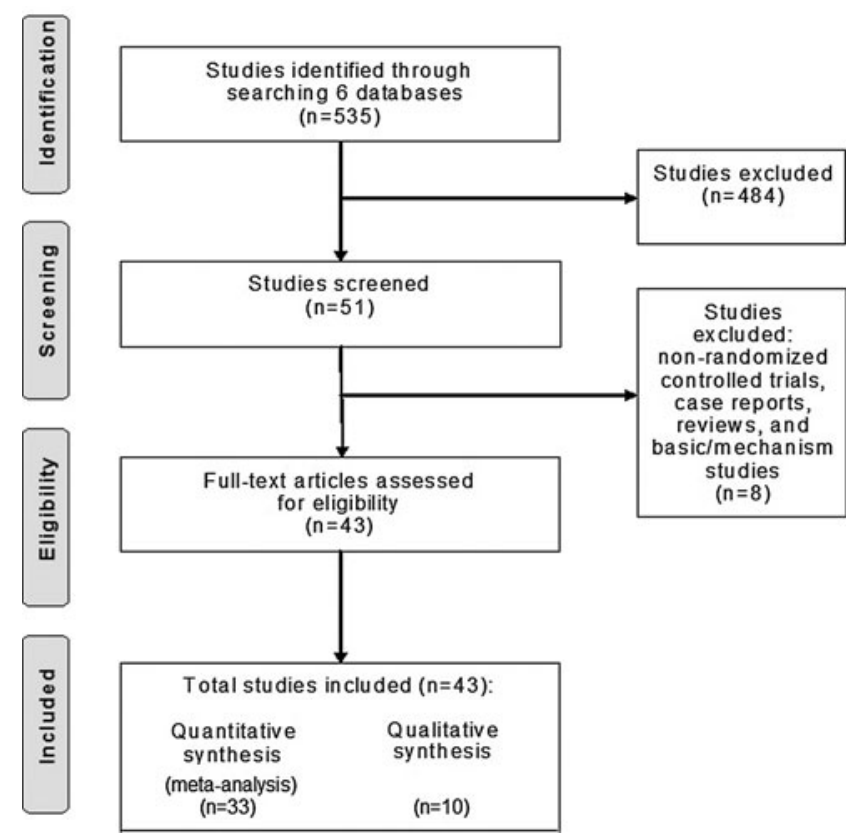

FIG. 1. Search strategy flow chart.

moxibustion, and combination of acupoint-stimulation therapies and herbal medicine. Controls included pharmaceutical medications and herbal medicine alone. Courses of treatment ranged from 1 to 12 weeks.

Degree of reduction in clinical symptoms (known as the cure rate) as the major outcome measurement was reported in all forty-three included trials. Responses to interventions were classified as cure, markedly effective, effective, and ineffective. ${ }^{53}$ Cure was defined as lesions totally faded (or $>95 \%$ faded) and only mild pigmentation and scars remaining. Markedly effective was defined as lesions faded $>60 \%$ and severity of lesions was alleviated. Effective was defined as lesions faded $20 \%-59 \%$ and severity of lesions was reduced. Ineffective was defined as lesions faded $<20 \%$ or worsening of lesions. Four trials ${ }^{13,19,43,45}$ counted the number of skin lesions, three trials ${ }^{14,42,45}$ reported QoL scores, two trials ${ }^{42,43}$ reported recurrence rates, and three trials ${ }^{14,42,43}$ listed laboratory test results.

\section{Methodological Quality}

According to the current authors' predefined qualityassessment criteria, all forty-three trials were evaluated as having a high risk of bias (Fig. 2). Study sample size varied from 20 to 112 participants, with an average of 40 patients per group. None of the trials reported sample-size calculation methodology. Fifteen trials $^{11,12,14,15,19,21,26,30,32,35,42,44-46,48}$ described randomization procedures, using random number tables or computer generation of random numbers, but only 1 trial $^{14}$ reported adequate allocation concealment. The majority of trials 
Table 1. Characteristics of 43 Included Trials

\begin{tabular}{|c|c|c|c|c|c|c|c|c|}
\hline \multirow{2}{*}{$\begin{array}{l}\text { Study 1st } \\
\text { author \& ref. }\end{array}$} & \multirow{2}{*}{$\begin{array}{c}\text { Diagnostic } \\
\text { criteria }\end{array}$} & \multicolumn{2}{|c|}{ Sample size $(M / F)$} & \multicolumn{2}{|c|}{ Sample age (range, $y$ ) } & \multicolumn{2}{|c|}{ Disease duration (average) } & \multirow{2}{*}{$\begin{array}{c}\text { Baseline } \\
\text { data } \\
\text { comparable? }\end{array}$} \\
\hline & & $I$ & $C$ & $I$ & $C$ & $I$ & $C$ & \\
\hline Chen $2007^{10}$ & $\begin{array}{l}\text { Chinese } \\
\text { criteria \& } \\
\text { Western } \\
\text { criteria }\end{array}$ & $10 / 26$ & $8 / 22$ & 22.13 & 21.63 & $2.61 \mathrm{y}$ & $1.96 \mathrm{y}$ & Yes \\
\hline Chen $2009^{11}$ & $\begin{array}{c}\text { Nonstandard } \\
\text { Chinese } \\
\text { criteria }\end{array}$ & $30 *$ & $60 *$ & $22.93 \pm 6.03$ & $21.83 \pm 6.07$ & $4.31 \pm 3.24 \mathrm{y}$ & $2.60 \pm 1.92 \mathrm{y}$ & Yes \\
\hline Cheng $2010^{12}$ & $\begin{array}{l}\text { Chinese } \\
\text { criteria }^{56}\end{array}$ & $27 / 23$ & $26 / 24$ & $21.2(14-35)$ & $21.5(13-36)$ & $6.5 \mathrm{~m}(1 \mathrm{w}-5 \mathrm{y})$ & $6.3 \mathrm{~m}(1 \mathrm{w}-6 \mathrm{y})$ & Yes \\
\hline Fan $2010^{13}$ & $\begin{array}{l}\text { Chinese } \\
\text { criteria }^{53}\end{array}$ & $15 / 10$ & $11 / 15$ & $22.24 \pm 4.087$ & $22.32 \pm 4.534$ & $1 \mathrm{y}-3 \mathrm{y}$ & $1 y-3 y$ & Yes \\
\hline Gong $2005^{14}$ & $\begin{array}{l}\text { Chinese } \\
\text { criteria }^{53}\end{array}$ & $9 / 11$ & $6 / 14$ & 21.3 & 21.5 & $2.89 \mathrm{y}$ & $3.57 \mathrm{y}$ & Yes \\
\hline Han $2010^{15}$ & $\begin{array}{c}\text { Nonstandard } \\
\text { Chinese } \\
\text { criteria }\end{array}$ & $18 / 28$ & $14 / 33$ & 25.83 & 24.68 & $2.35 \mathrm{y}$ & $2.15 \mathrm{y}$ & Yes \\
\hline He $2009^{16}$ & $\begin{array}{l}\text { Chinese } \\
\text { criteria }^{54}\end{array}$ & $8 / 16$ & $10 / 12$ & $25.2(17-41)$ & $23.6(16-38)$ & $20 \mathrm{~d}-16 \mathrm{y}$ & $1 \mathrm{~m}-17 \mathrm{y}$ & Yes \\
\hline
\end{tabular}


Table 1. (Continued)

\begin{tabular}{|c|c|c|c|c|}
\hline Intervention & Control & $\begin{array}{l}\text { Duration of } \\
\text { treatment }(w)\end{array}$ & Outcome & Adverse events \\
\hline $\begin{array}{l}\text { Warm needling \& } \\
\text { moxibustion at: CV 4, CV } \\
\text { 6, ST 36, BL 20, \& BL 23; } \\
\text { additional points according } \\
\text { to syndrome } \\
\text { differentiation, } 30 \text { minutes, } \\
1 \times / 2 \text { d }\end{array}$ & $\begin{array}{l}\text { Acupuncture applied to } \\
\text { same acupoints as for } \\
\text { treatment group, } 1 \times / \\
\text { every other d }\end{array}$ & 9 & $\begin{array}{l}\text { Response to } \\
\text { intervention }\end{array}$ & $\begin{array}{l}\text { Yes ( } 3 \text { patients in } \\
\text { intervention group had } \\
\text { dry stools, thirst, } \\
\text { irritability) }\end{array}$ \\
\hline $\begin{array}{l}\text { Bloodletting, followed by } \\
\text { cupping at BL } 13 \& \text { BL } 21 \text {, } \\
\text { combined with body } \\
\text { acupuncture } 2 \times / w ; \text { herbal } \\
\text { medicine, twice per d }\end{array}$ & Herbal medicine $2 \times / d$ & 4 & $\begin{array}{l}\text { Response to } \\
\text { intervention }\end{array}$ & No \\
\hline $\begin{array}{l}\text { Bai Xian Xia Ta Re tablet, } 5 \\
\text { pills (adolescents, } 3 \text { pills), } \\
3 \times / \mathrm{d} \text {; ear acupressure with } \\
\text { vaccaria seed; main points: } \\
\text { CO } 14 \text {, CO 4, LO } 5,6 \mathrm{i} \text {; } \\
\text { additional points according } \\
\text { to syndrome } \\
\text { differentiation; patients } \\
\text { instructed to press } 1-2 \text { min } \\
1 \times / \mathrm{w}\end{array}$ & $\begin{array}{l}\text { Bai Xian Xia Ta Re tablet, } \\
5 \text { pills (adolescents, } 3 \\
\text { pills) } 3 \times / d\end{array}$ & 4 & $\begin{array}{l}\text { Response to } \\
\text { intervention }\end{array}$ & NR \\
\hline $\begin{array}{l}\text { Fire needling \& acupuncture } \\
\text { on the lesion area, } \\
1 \times / \text { every } 5 \mathrm{~d}\end{array}$ & $\begin{array}{l}\text { Tazarotene cream applied } \\
\text { to lesions at bedtime } \\
\text { every night, } \\
\text { clindamycin gel applied } \\
\text { to lesions } 1 \times / \text { every } \\
\text { morning }\end{array}$ & 3 & $\begin{array}{l}\text { Skin-lesion count; } \\
\text { response to } \\
\text { intervention; } \\
\text { adverse effects }\end{array}$ & $\begin{array}{l}\text { Yes (24 cases in acu } \\
\text { group \& } 9 \text { cases in } \\
\text { control group had pain; } \\
24 \text { cases in acu group \& } \\
6 \text { cases in control group } \\
\text { had redness \& swelling } \\
\text { of portions of lesion } \\
\text { area; } 13 \text { cases in acu } \\
\text { group \& } 15 \text { cases in } \\
\text { control had itching in } \\
\text { acupoint areas) }\end{array}$ \\
\hline $\begin{array}{l}\text { Acupuncture on skin-lesion } \\
\text { area, } 30 \text { min } 1 \times / \text { every } 2 \text {; } \\
\text { ear acupressure with } \\
\text { vaccaria seed on CO } 18 \text {, } \\
\text { TG } 2 \mathrm{p}, \text { AH } 6 \text { a CO } 14 \& \\
\text { LO } 5,6 \mathrm{i} \text {; patients instructed } \\
\text { to press } 3-5 \text { min } 2 \times / d\end{array}$ & $\begin{array}{l}\text { Licorsinc capsule, } 0.25 \mathrm{~g} \text {, } \\
\quad 3 \times / \mathrm{d}\end{array}$ & 4 & $\begin{array}{l}\text { Laboratory tests; } \\
\text { QoL-Acne; } \\
\text { GAGS; response } \\
\text { to intervention }\end{array}$ & NR \\
\hline $\begin{array}{l}\text { Acupuncture of abdominal } \\
\text { acupoints: CV 12, CV } 10 \text {, } \\
\text { CV } 6, \text { CV } 4, \text { ST } 24, \text { ST } 26 \text {, } \\
\text { KI } 13 \text { for } 30 \text { min, } 3 \times / \mathrm{w} \text {, } \\
1 \times / \text { every } 2 \text { days }\end{array}$ & $\begin{array}{l}\text { Isotretinoin, } 10 \mathrm{mg}, 2 \times / \mathrm{d} \\
\text { for } 1 \mathrm{~m} \text {, then } 10 \mathrm{mg} \\
1 \times / \mathrm{d}\end{array}$ & 8 & $\begin{array}{l}\text { Response to } \\
\text { intervention }\end{array}$ & NR \\
\hline $\begin{array}{l}\text { Acupuncture of abdominal } \\
\text { acupoints: CV 12, CV 10, }\end{array}$ & $\begin{array}{l}\text { Topical metronidazole } \\
\text { solution } 2 \times / \mathrm{d}\end{array}$ & 3 & $\begin{array}{l}\text { Response to } \\
\text { intervention }\end{array}$ & NR \\
\hline
\end{tabular}
CV 6, CV 4 ST 24, ST 26; facial acupoints EX-HN 3, "ouch" point $30 \mathrm{~min}$; TDP mineral lamp 15-20 min $1 \times / \mathrm{d}$ for $7 \mathrm{~d}$, then $1 \times /$ every $2 \mathrm{~d}$

\section{Acupuncture applied to same acupoints as for treatment group, $1 \times 1$ ery other $d$}

ai Xian Xia Ta Re tablet, 5 pills (adolescents, 3 pills) $3 \times / d$

zarotene cream applied every night, clindamycin gel applied to lesions $1 \times$ /every morning solution $2 \times / d$
Yes ( 3 patients in intervention group had dry stools, thirst, itability)

Yes (24 cases in acu control group had pain; 24 cases in acu group \& 6 cases in control group redness \& swelling ons of lesion group \& 15 cases in control had itching in NR

QoL-Acne;

GAGS; response

to intervention intervention 
Table 1. (Continued)

\begin{tabular}{|c|c|c|c|c|c|c|c|c|}
\hline \multirow{2}{*}{$\begin{array}{l}\text { Study 1st } \\
\text { author \& ref. }\end{array}$} & \multirow{2}{*}{$\begin{array}{l}\text { Diagnostic } \\
\text { criteria }\end{array}$} & \multicolumn{2}{|c|}{ Sample size $(M / F)$} & \multicolumn{2}{|c|}{ Sample age (range, $y$ ) } & \multicolumn{2}{|c|}{ Disease duration (average) } & \multirow{2}{*}{$\begin{array}{c}\text { Baseline } \\
\text { data } \\
\text { comparable? }\end{array}$} \\
\hline & & $I$ & C & $I$ & C & $I$ & C & \\
\hline Hong $2011^{17}$ & $\begin{array}{l}\text { Nonstandard } \\
\text { Chinese } \\
\text { criteria }\end{array}$ & $8 / 11$ & $7 / 11)$ & $24(15-36)$ & $23(14-35)$ & $2.5(1-10) \mathrm{y}$ & $2.2(1-11) \mathrm{y}$ & Yes \\
\hline Hou $2009^{18}$ & $\begin{array}{l}\text { Chinese } \\
\text { criteria }^{55}\end{array}$ & $21 / 24$ & $18 / 24$ & $16-38$ & $17-38$ & $2 \mathrm{~m}-5 \mathrm{y}$ & $3 \mathrm{~m}-4 \mathrm{y}$ & NR \\
\hline Huang $2011^{19}$ & $\begin{array}{l}\text { Chinese } \\
\text { criteria }^{54}\end{array}$ & $7 / 23$ & $7 / 23$ & $19.23 \pm 3.05$ & $19.03 \pm 3.18$ & $11.57 \mathrm{~d} \pm 7.55 \mathrm{~d}$ & $11.47 \mathrm{~d} \pm 6.72 \mathrm{~d}$ & Yes \\
\hline Huang $2010^{20}$ & $\begin{array}{l}\text { Nonstandard } \\
\text { Chinese } \\
\text { criteria }\end{array}$ & $76^{*}$ & $50^{*}$ & $23(1$ & 5-35) & & $-5 \mathrm{y}$ & Yes \\
\hline Huang $2009^{21}$ & $\begin{array}{l}\text { Chinese } \\
\text { criteria }^{54}\end{array}$ & $12 / 18$ & $10 / 20$ & 15 & 45 & & $-2 \mathrm{y}$ & Yes \\
\hline Huang $2011^{22}$ & NR & $14 / 16$ & $15 / 15$ & 21.3 & 19.9 & $24.7 \mathrm{~m}$ & $26.0 \mathrm{~m}$ & Yes \\
\hline Jin $2009^{23}$ & NR & $14 / 18$ & $12 / 14$ & $24.5(18-24)$ & $23(16-30)$ & $(4.7 \pm 1.2) \mathrm{y}$ & $(4.8 \pm 1.14) \mathrm{y}$ & Yes \\
\hline Lan $2004^{24}$ & $\begin{array}{l}\text { Chinese } \\
\text { criteria } \\
\text { without } \\
\text { source }\end{array}$ & $0 / 38$ & $0 / 34$ & $\mathrm{~N}$ & & & $\mathrm{JR}$ & Yes \\
\hline Li $1995^{25}$ & NR & $35 / 21$ & $25 / 15$ & $\mathrm{~N}$ & & & $-6 y$ & NR \\
\hline
\end{tabular}


Table 1. (Continued)

\begin{tabular}{|c|c|c|c|c|}
\hline Intervention & Control & $\begin{array}{l}\text { Duration of } \\
\text { treatment }(w)\end{array}$ & Outcome & Adverse events \\
\hline $\begin{array}{l}\text { Bloodletting at HX 6,7i, GV } \\
\text { 14, BL 13, BL } 21 \& \text { BL } 20 \\
3-5 \times \text {; cupping on GV } 14 \text {, } \\
\text { BL } 13 \text {, BL } 21 \& \text { BL } 20 \text { for } \\
10-15 \text { min } 2 \times / w \text {; Chinese } \\
\text { medicinal facial mask } \\
2 \times / \mathrm{w}\end{array}$ & $\begin{array}{l}\text { Chinese medicinal facial } \\
\text { mask } 2 \times / \mathrm{w}\end{array}$ & 5 & $\begin{array}{l}\text { Response to } \\
\text { intervention }\end{array}$ & NR \\
\hline $\begin{array}{l}\text { Bloodletting, followed by } \\
\text { cupping at GV 14, BL } 28, \\
\text { BL 25, BL } 21 \& \text { BL } 13 \text {; } \\
\text { Blood Stasis point, } \\
1 \times / \text { every } 2 \text { d; ear acupoint } \\
\text { needle embedding } 3-5 \text { d at } \\
\text { acupoints TF } 4 \text {, TF } 2, \mathrm{CO} \\
18 \text { \& TG } 2\end{array}$ & $\begin{array}{l}\text { Bloodletting, followed by } \\
\text { cupping at GV } 14, \mathrm{BL} \\
28, \mathrm{BL} 25, \mathrm{BL} 21 \& \mathrm{BL} \\
13 \text {; Blood Stasis point } \\
1 \times \text { /every } 2 \mathrm{~d}\end{array}$ & 2 & $\begin{array}{l}\text { Response to } \\
\text { intervention }\end{array}$ & NR \\
\hline $\begin{array}{l}\text { Acupuncture at "ouch" } \\
\text { point, } 30 \text { min } 1 \times / \text { every } 2 \\
\text { d, } 3 \times / \mathrm{w} \text {; Pi Pa Qing Fei } \\
\text { decoction, } 250 \mathrm{~mL}, 2 \times / \mathrm{d} \text {; } \\
\text { Chinese herbal facial } \\
\text { mask, } 30 \text { min } 1 \times / \mathrm{d}\end{array}$ & $\begin{array}{l}\text { Pi Pa Qing Fei decoction, } \\
250 \mathrm{~mL}, 2 \times / \mathrm{d} \text {; Chinese } \\
\text { herbal facial mask, } \\
30 \mathrm{~min} 1 \times / \mathrm{d}\end{array}$ & 4 & $\begin{array}{l}\text { Skin-lesion count; } \\
\text { response to } \\
\text { intervention; } \\
\text { adverse effects }\end{array}$ & $\begin{array}{l}\text { Yes (6 patients in acu } \\
\text { group reported black \& } \\
\text { blue of some skin areas } \\
\text { after acupuncture) }\end{array}$ \\
\hline $\begin{array}{l}\text { Bloodletting, followed by } \\
\text { cupping at GV10, GV14, } \\
\text { BL13, BL15, BL20 for } \\
5-10 \text { min } 2 \times / w \text {; herbal } \\
\text { preparation, } 50 \mathrm{~mL}, 3 \times / \mathrm{d} \text {; } \\
\text { topical facial cream }\end{array}$ & $\begin{array}{l}\text { Herbal preparation } 50 \mathrm{~mL} \text {, } \\
3 \times / \mathrm{d} \text {; topical facial } \\
\text { cream }\end{array}$ & 4 & $\begin{array}{l}\text { Response to } \\
\text { intervention }\end{array}$ & NR \\
\hline $\begin{array}{l}\text { Autologous blood injection at } \\
\text { acupoints LI } 11 \& \text { BL } 13 \text {; } \\
\text { EA at skin-lesion area for } \\
30 \mathrm{~min}, 3 \times / \mathrm{w}\end{array}$ & $\begin{array}{l}\text { Autologous blood } \\
\text { injection at acupoints } \\
\text { LI } 11 \& \text { BL } 13 \text { for } \\
30 \mathrm{~min}, 3 \times / \mathrm{w}\end{array}$ & 4 & $\begin{array}{l}\text { Response to } \\
\text { intervention }\end{array}$ & NR \\
\hline $\begin{array}{l}\text { Autologous blood injection, } \\
4 \mathrm{~mL} \text {, at bilateral acupoint } \\
\text { ST } 36,1 \times / \mathrm{w}\end{array}$ & $\begin{array}{l}\text { Viaminate capsules, } \\
0.025 \mathrm{~g}, 3 \times / \mathrm{d} \\
\text { tetracycline, } 0.25 \mathrm{~g} \\
3 \times / \mathrm{d}\end{array}$ & 3 & $\begin{array}{l}\text { Response to } \\
\text { intervention }\end{array}$ & NR \\
\hline $\begin{array}{l}\text { Body \& facial acupuncture } \\
30 \text { min once every } 2 \text { days; } \\
\text { herbal facial mask applied } \\
\text { for } 20 \text { min } 1 \times / \text { every } 2-3 \mathrm{~d}\end{array}$ & $\begin{array}{l}\text { Herbal facial mask } \\
\text { applied } 20 \text { min once } \\
\text { every } 2-3 \mathrm{~d}\end{array}$ & 4 & $\begin{array}{l}\text { Response to } \\
\text { intervention }\end{array}$ & NR \\
\hline $\begin{array}{l}\text { Acupuncture at lesion area, } \\
\text { LI 4, SP } 36,30 \mathrm{~min} 3 \times / \mathrm{w} \text {, } \\
3 \text { w/cycle for } 2 \text { cycles; } \\
\text { Qing Shen Fen } \mathrm{Ci} \text { Qing } \\
\text { oral liquid ,20 mL, } 3 \times / \mathrm{d}\end{array}$ & $\begin{array}{l}\text { Qin Shen Fen Ci Qing } \\
\text { oral liquid, } 20 \mathrm{~mL} \text {, } \\
3 \times / \mathrm{d} \text {. }\end{array}$ & 6 & $\begin{array}{l}\text { Response to } \\
\text { intervention; } \\
\text { adverse events }\end{array}$ & NR \\
\hline $\begin{array}{l}\text { Use point-detection device to } \\
\text { test/stimulate ear points } \\
\text { TG } 2 \mathrm{p}, \mathrm{CO} 7, \mathrm{CO} 14 \& \mathrm{CO} \\
4 \text { for } 30 \mathrm{~min} \text { for } 1 \mathrm{~m} ; \\
\text { herbal decoction } 1 \times / \mathrm{d} \\
\text { for } 1 \mathrm{~m}\end{array}$ & $\begin{array}{l}\text { Herbal decoction } 1 \times / \mathrm{d} \\
\quad \text { for } 1 \mathrm{~m}\end{array}$ & 4 & $\begin{array}{l}\text { Response to } \\
\text { intervention }\end{array}$ & NR \\
\hline
\end{tabular}


Table 1. (Continued)

\begin{tabular}{|c|c|c|c|c|c|c|c|c|}
\hline \multirow{2}{*}{$\begin{array}{l}\text { Study 1st } \\
\text { author \& ref. }\end{array}$} & \multirow{2}{*}{$\begin{array}{c}\text { Diagnostic } \\
\text { criteria }\end{array}$} & \multicolumn{2}{|c|}{ Sample size $(M / F)$} & \multicolumn{2}{|c|}{ Sample age (range, y) } & \multicolumn{2}{|c|}{ Disease duration (average) } & \multirow{2}{*}{$\begin{array}{c}\text { Baseline } \\
\text { data } \\
\text { comparable? }\end{array}$} \\
\hline & & $I$ & $C$ & $I$ & $C$ & $I$ & $C$ & \\
\hline Li $2009^{26}$ & $\begin{array}{l}\text { Chinese } \\
\text { criteria }^{53}\end{array}$ & $0 / 29$ & $0 / 30$ & $25.48 \pm 4.09$ & $25.57 \pm 4.67$ & $<13 \mathrm{y}$ & $<13 y$ & NR \\
\hline
\end{tabular}

\begin{tabular}{|c|c|c|c|c|c|c|c|c|}
\hline Li $2011^{27}$ & NR & $48^{*}$ & $48 *$ & 22.8 & 4-32) & $22 \mathrm{~m}($ & $-10 \mathrm{y})$ & Yes \\
\hline Li $2011^{28}$ & $\begin{array}{l}\text { Chinese } \\
\text { criteria }^{53}\end{array}$ & $10 / 13$ & $10 / 12$ & $25.3(18-39)$ & $24.5(18-35)$ & $19.17(1-65 \mathrm{~m})$ & $19.36(2-67 \mathrm{~m})$ & NR \\
\hline Liu $2008^{29}$ & NR & $60 *$ & $60^{*}$ & \multicolumn{2}{|c|}{$14-41$} & \multicolumn{2}{|c|}{$2 \mathrm{~m}-10 \mathrm{y}$} & Yes \\
\hline
\end{tabular}

\begin{tabular}{|c|c|c|c|c|c|c|c|}
\hline Liu $2009^{30}$ & $\begin{array}{l}\text { Chinese } \\
\text { Criteria \& } \\
\text { Western } \\
\text { criteria }\end{array}$ & $14 / 29$ & $39 *$ & 23.6 & 24.1 & $2 \mathrm{w}-5 \mathrm{y}$ & $3 \mathrm{~m}-4 \mathrm{y}$ \\
\hline
\end{tabular}

\begin{tabular}{|c|c|c|c|c|c|c|c|}
\hline $\mathrm{Lu} 2011^{31}$ & $\begin{array}{l}\text { Nonstandard } \\
\text { Chinese } \\
\text { criteria }\end{array}$ & $21 / 39$ & $18 / 42$ & $\begin{array}{c}20.17 \pm 4.48 \\
\quad(13-34)\end{array}$ & $\begin{array}{c}22.17 \pm 4.12 \\
\quad(14-30)\end{array}$ & $1 \mathrm{~m}-10 \mathrm{y}$ & $1 \mathrm{~m}-10 \mathrm{y}$ \\
\hline
\end{tabular}

$\begin{array}{cccccccr}\text { Mao 2008 } & \begin{array}{c}\text { Chinese } \\ \text { criteria }^{54}\end{array} & 16 / 25 & 43 * & 16-21 & 1 \mathrm{w}-2 \mathrm{~m} & 1 \mathrm{w}-3 \mathrm{~m} & \text { Yes } \\ \text { Mi } 2011^{33} & \begin{array}{c}\text { Chinese } \\ \text { criteria }^{53}\end{array} & 14 / 16 & 18 / 12 & 22.31(31-32) & 25.27(16-30) & \text { NR } & \text { Yes }\end{array}$


Table 1. (Continued)

\begin{tabular}{|c|c|c|c|c|}
\hline Intervention & Control & $\begin{array}{l}\text { Duration of } \\
\text { treatment }(w)\end{array}$ & Outcome & Adverse events \\
\hline $\begin{array}{l}\text { Herbal decoction, } 50 \mathrm{~mL} \text {, } \\
2 \times / \mathrm{d} \text {; Cuo Ling Ding } \\
\text { applied topically to } \\
\text { lesions; acupoint injection } \\
\text { with } 1 \mathrm{~mL} \text { of danshen } \\
\text { extract at ST } 36,1 \times / \text { every } \\
3-5 \mathrm{~d} \text {; ear acupuncture at } \\
\text { TF } 4 \text {, TG } 2 \text { p, AH } 6 \text { a \& CO } \\
18,1 \times / \text { every } 7 \mathrm{~d}\end{array}$ & $\begin{array}{l}\text { Herbal decoction, } 50 \mathrm{~mL} \text {, } \\
2 \times / \mathrm{d} \text {; Cuo Ling Ding } \\
\text { applied topically to } \\
\text { lesions; acupoint } \\
\text { injection with } 1 \mathrm{~mL} \text { of } \\
\text { danshen at ST } 36 \text {, } \\
1 \times / \text { every } 3-5 \mathrm{~d}\end{array}$ & 4 & $\begin{array}{l}\text { Response to } \\
\text { intervention }\end{array}$ & NR \\
\hline $\begin{array}{l}\text { Acupuncture at LI 4, SI } 3 \text { \& } \\
\text { PC 8; herbal decoction; } \\
\text { She Dan cream applied } \\
\text { topically to lesions }\end{array}$ & $\begin{array}{l}\text { Herbal decoction; She } \\
\text { Dan Shuan applied } \\
\text { topically to lesions }\end{array}$ & 12 & $\begin{array}{l}\text { Response to } \\
\text { intervention }\end{array}$ & No \\
\hline $\begin{array}{l}\text { Fire needling at "ouch" } \\
\text { point, BL } 13, \mathrm{BL} 15, \mathrm{BL} \\
18, \mathrm{BL} 23, \mathrm{CV} 4, \mathrm{CV} 6, \\
\mathrm{CV} 12 \& \mathrm{~S} 251 \times / \mathrm{w} \text { for } 8 \\
\text { w; Yang He decoction }\end{array}$ & Yang He decoction & 8 & $\begin{array}{l}\text { Response to } \\
\text { intervention }\end{array}$ & NR \\
\hline $\begin{array}{l}\text { Autologous blood injection, } \\
5 \mathrm{~mL} \text {, injection at bilateral } \\
\text { ST } 36,1 \times / \mathrm{w} \text {; medications } \\
\text { same as control group }\end{array}$ & $\begin{array}{l}\text { Vitamin B tablet, zinc } \\
\text { gluconate tablet, Luo } \\
\text { Hong enzyme capsule, } \\
\text { Pi Pa Qing Fei } \\
\text { decoction; topical acne } \\
\text { tincture; skin hygiene; } \\
\text { Qing } \mathrm{Da} \text { enzyme } \\
\text { injection or } \\
\text { triamcinolone injection } \\
1 \times / \mathrm{w}\end{array}$ & 6 & $\begin{array}{l}\text { Response to } \\
\text { intervention }\end{array}$ & NR \\
\hline $\begin{array}{l}\text { Acupuncture for } 30 \text { min, } \\
\text { followed by flash cupping } \\
\text { mainly at ST } 3 \text {, ST } 4, \text { ST } 6 \text {, } \\
\text { ST } 7 \text { \& SI } 18 ; 10 \text { sessions } \\
\text { per course, } 2 \text { courses total } \\
(1 \times / \text { d for the first course, } \\
\text { every other day for the } \\
\text { second course) }\end{array}$ & $\begin{array}{l}\text { Acupuncture mainly at ST } \\
3, \text { ST } 4, \text { ST } 6, \text { ST } 7 \& \\
\text { SI } 18,10 \text { sessions per } \\
\text { course, } 2 \text { courses total } \\
(1 \times / d \text { for the first } \\
\text { course, every other d } \\
\text { for the second course })\end{array}$ & 4 & $\begin{array}{l}\text { Response to } \\
\text { intervention }\end{array}$ & No \\
\hline $\begin{array}{l}\text { Flash cupping at DU } 14 \text {, } \\
\text { followed by needling for } \\
5-10 \text { min } \& \text { cupping for } \\
5 \text { min, } 1 \times / \text { every } 2-3 \text { days; } \\
\text { herbal decoction } 2 \times / d\end{array}$ & Herbal preparation $2 \times / d$ & 4 & $\begin{array}{l}\text { Response to } \\
\text { intervention }\end{array}$ & No \\
\hline $\begin{array}{l}\text { Acupuncture at Ex-B } 2, \mathrm{ST} \\
36 \& \text { LI } 4 \text { for } 15-30 \mathrm{~min}, \\
1 \times / \mathrm{d} \text { for } 5 \mathrm{~d}\end{array}$ & $\begin{array}{l}\text { Oral minocyclin, } 50 \mathrm{mg} \text {, } \\
2 \times / \mathrm{d}\end{array}$ & 1 & $\begin{array}{l}\text { Response to } \\
\text { intervention }\end{array}$ & NR \\
\hline $\begin{array}{l}\text { Acupuncture at CV } 12, \mathrm{CV} \\
10, \mathrm{CV} 4, \mathrm{ST} 26, \mathrm{ST} 25 \text { or } \\
30 \mathrm{~min}, 1 \times / \text { every } 2 \mathrm{~d}, 1 \mathrm{~m} / \\
\text { course for } 2 \text { courses; warm } \\
\text { needling at skin lesion } \\
\text { area; acupoint injection } \\
\text { with danshen injection at } \\
\text { ST } 36 \& \text { LI } 11\end{array}$ & $\begin{array}{l}\text { Warm needling at skin } \\
\text { lesion area; acupoint } \\
\text { injection with danshen } \\
\text { injection at ST } 36 \text { \& LI } \\
11\end{array}$ & 8 & $\begin{array}{l}\text { Response to } \\
\text { intervention }\end{array}$ & NR \\
\hline
\end{tabular}


Table 1. (Continued)

\begin{tabular}{|c|c|c|c|c|c|c|c|c|}
\hline \multirow{2}{*}{$\begin{array}{l}\text { Study 1st } \\
\text { author \& ref. }\end{array}$} & \multirow{2}{*}{$\begin{array}{c}\text { Diagnostic } \\
\text { criteria }\end{array}$} & \multicolumn{2}{|c|}{ Sample size $(M / F)$} & \multicolumn{2}{|c|}{ Sample age (range, $y$ ) } & \multicolumn{2}{|c|}{ Disease duration (average) } & \multirow{2}{*}{$\begin{array}{c}\text { Baseline } \\
\text { data } \\
\text { comparable? }\end{array}$} \\
\hline & & $I$ & C & $I$ & C & $I$ & C & \\
\hline Song $2011^{34}$ & $\begin{array}{l}\text { Nonstandard } \\
\text { Chinese } \\
\text { criteria }\end{array}$ & $34 / 26$ & $36 / 24$ & $20.3(14-30)$ & $21.1(13-31)$ & $389 \mathrm{~d}(7 \mathrm{~d}-3 \mathrm{y})$ & 392 d (5 d-3 y) & Yes \\
\hline Wang $2011^{35}$ & $\begin{array}{l}\text { Chinese } \\
\text { criteria }^{53}\end{array}$ & $10 . / 14$ & $11 / 13$ & $22.58(18-32)$ & $22.33\left(18 \_33\right)$ & $>2 \mathrm{w}$ & $>2 \mathrm{w}$ & Yes \\
\hline Wang $2009^{36}$ & $\begin{array}{l}\text { Chinese } \\
\text { criteria } \\
\text { without } \\
\text { source }\end{array}$ & $35 / 33$ & $30 / 30$ & 26.5 & 25 & $6 \mathrm{~m}-7 \mathrm{y}$ & $5 \mathrm{~m}-8 \mathrm{y}$ & Yes \\
\hline Wang $2007^{37}$ & $\begin{array}{l}\text { Nonstandard } \\
\text { Chinese } \\
\text { criteria }\end{array}$ & $30 *$ & $30 *$ & $18 \mathrm{~d}-$ & $-37 d$ & 10 & -6 y & Yes \\
\hline Wu $2010^{38}$ & NR & $30^{*}$ & $28^{*}$ & $16-25$ & $15-25$ & $4 \mathrm{~m}-6 \mathrm{y}$ & $3 \mathrm{~m}-6 \mathrm{y}$ & NR \\
\hline Wu $1998^{39}$ & NR & $34 *$ & $34 *$ & 19.5 & 16-24) & & $-4 y$ & NR \\
\hline Wu $2008^{40}$ & $\begin{array}{c}\text { Chinese } \\
\text { criteria } \\
\text { without } \\
\text { source }\end{array}$ & $9 / 21$ & $7 / 23$ & $26.13 \pm 2.54$ & $24.83 \pm 3.07$ & $15.20 \mathrm{w} \pm 136 \mathrm{w}$ & $14.53 w+187 w$ & Yes \\
\hline Xie $2009^{41}$ & $\begin{array}{l}\text { Chinese } \\
\text { criteria } \\
\text { without } \\
\text { source }\end{array}$ & $16 / 22$ & $18 / 12$ & $15-38$ & $14-36$ & & -9 y & Yes \\
\hline
\end{tabular}


Table 1. (Continued)

\begin{tabular}{|c|c|c|c|c|}
\hline Intervention & Control & $\begin{array}{l}\text { Duration of } \\
\text { treatment }(w)\end{array}$ & Outcome & Adverse events \\
\hline $\begin{array}{l}\text { Bloodletting, followed by } \\
\text { cupping at GV 14, BL 13, } \\
\text { BL 18, BL } 20 \text { \& BL21 }\end{array}$ & $\begin{array}{l}\text { Vitamin B tablet, oral } \\
\text { metronidazole, or oral } \\
\text { erythromycin; topical } \\
\text { metronidazole } 1 \%-5 \% \text {, } \\
\text { cream; topical benzoyl } \\
\text { peroxide gel; topical } \\
\text { sulfur preparation; } \\
\text { topical Mei Lu Xiao } \\
\text { Cuo cream }\end{array}$ & 4 & $\begin{array}{l}\text { Response to } \\
\text { intervention }\end{array}$ & NR \\
\hline $\begin{array}{l}\text { Acupuncture \& guasha at GB } \\
14, \text { SI } 18, \text { GV } 14, \text { LI } 4, \mathrm{LI} \\
11 \text { \& S } 441 \times / \text { every } 2 \mathrm{~d} \text {; } \\
\text { scraping the first lateral of } \\
\text { BL meridian, once per w }\end{array}$ & $\begin{array}{l}\text { Scraping the first lateral of } \\
\text { BL meridian, once } \\
\text { per w }\end{array}$ & 4 & $\begin{array}{l}\text { Response to } \\
\text { intervention }\end{array}$ & NR \\
\hline $\begin{array}{l}\text { Acupoint catgut embedding } \\
\text { at BL } 13 \& \text { ST } 36 \text { as main } \\
\text { points; additional points } \\
\text { selected according to } \\
\text { syndrome differentiation, } \\
1 \times / \mathrm{m} \text {; oral herbal } \\
\text { decoction }\end{array}$ & Oral herbal decoction & 8 & $\begin{array}{l}\text { Response to } \\
\text { intervention }\end{array}$ & $\begin{array}{l}\text { Yes (distending pain } \\
\text { during the acupoint } \\
\text { catgut embedding) }\end{array}$ \\
\hline $\begin{array}{l}\text { Acupuncture \& } \\
\text { moxibustion }+ \text { acupoint } \\
\text { bloodletting \& moving } \\
\text { cupping (acupuncture for } \\
30 \text { min, } 1 \times / d \text {; cupping for } \\
5-10 \text { min, } 1 \times / \text { every } 3 \mathrm{~d} \text {; } \\
10 \text { sessions as } 1 \text { course }\end{array}$ & $\begin{array}{l}\text { Acupuncture } \& \\
\text { moxibustion, } 1 \times / d\end{array}$ & 4 & $\begin{array}{l}\text { Response to } \\
\text { intervention }\end{array}$ & NR \\
\hline $\begin{array}{l}\text { Bloodletting, followed by } \\
\text { cupping at BL } 13, \text { BL } 15 \text {, } \\
\text { BL } 18, \text { BL } 20, \text { BL } 21 \& \\
\text { BL } 23 \text { for } 10-15 \text { min, } \\
1 \times \text { /every } 5-7 \text { d }\end{array}$ & $\begin{array}{l}\text { Oral tanshinone } 1 \mathrm{~g}, 3 \times / \mathrm{d} \\
\quad \text { for } 3 \mathrm{w}\end{array}$ & 4 & $\begin{array}{l}\text { Response to } \\
\text { intervention }\end{array}$ & NR \\
\hline $\begin{array}{l}\text { Acupoint injection at L I4, LI } \\
11 \text {, SP } 6 \text { \& ST } 36 \text { of } \\
\text { extracts of yu xing cao \& } \\
\text { dang gui, } 4 \mathrm{~mL} \text { each, } \\
\text { alternating at } 1-2 \\
\text { acupoints, } 1 \times / \text { every } 2 \mathrm{~d}\end{array}$ & $\begin{array}{l}\text { Metronidazole tablet, } \\
0.2 \mathrm{~g}, 3 \times / \mathrm{d} \text {; } \\
\text { minocycline tablet, } \\
0.05 \mathrm{~g}, 2 \times / \mathrm{d}\end{array}$ & 3 & $\begin{array}{l}\text { Response to } \\
\text { intervention }\end{array}$ & NR \\
\hline $\begin{array}{l}\text { Bloodletting, followed by } \\
\text { cupping once daily at } \\
\text { "ouch" points, the BL } \\
\text { channel \& at BL } 13 \text { until } \\
7-8 \mathrm{~mL} \text { of blood is let }\end{array}$ & $\begin{array}{l}\text { Tetracycline, } 0.25 \mathrm{~g}, \\
4 \times / \mathrm{d} ; \text { ketoconazole, } \\
2 \% \text {,cream } 1 \times / \mathrm{d}\end{array}$ & 4 & $\begin{array}{l}\text { Response to } \\
\text { intervention }\end{array}$ & NR \\
\hline $\begin{array}{l}\text { Acupuncture at BL } 13, \mathrm{GV} \\
14, \mathrm{LI} 11, \mathrm{~L} \text { I4 \& SI } 18,\end{array}$ & $\begin{array}{l}\text { Medicinal facial mask } \\
30 \text { min } 1 \times / d\end{array}$ & 3 & $\begin{array}{l}\text { Response to } \\
\text { intervention }\end{array}$ & NR \\
\hline
\end{tabular}
with additional points BL 12, LU 11, ST 36 \& SP6 for $30 \mathrm{~min} 1 \times / \mathrm{d}$; medicinal facial mask for $30 \mathrm{~min} 1 \times / \mathrm{d}$

\begin{abstract}
Vitamin B tablet, oral metronidazole, or oral cream; topical benzoyl peroxide gel; topical sulfur preparation; topical Mei Lu Xiao uo cream BL meridian, once per $\mathrm{w}$
\end{abstract}

Oral herbal decoction

Acupuncture \& moxibustion, $1 \times / d$

Oral tanshinone $1 \mathrm{~g}, 3 \times / \mathrm{d}$ $30 \min 1 \times / d$ intervention 
Table 1. (Continued)

\begin{tabular}{|c|c|c|c|c|c|c|c|c|}
\hline \multirow{2}{*}{$\begin{array}{l}\text { Study 1st } \\
\text { author \& ref. }\end{array}$} & \multirow{2}{*}{$\begin{array}{c}\text { Diagnostic } \\
\text { criteria }\end{array}$} & \multicolumn{2}{|c|}{ Sample size $(M / F)$} & \multicolumn{2}{|c|}{ Sample age (range, y) } & \multicolumn{2}{|c|}{ Disease duration (average) } & \multirow{2}{*}{$\begin{array}{c}\text { Baseline } \\
\text { data } \\
\text { comparable? }\end{array}$} \\
\hline & & $I$ & $C$ & $I$ & $C$ & $I$ & $C$ & \\
\hline Xie $2011^{42}$ & $\begin{array}{l}\text { Chinese } \\
\text { criteria }^{55}\end{array}$ & $7 / 22$ & $6 / 22$ & $\begin{array}{c}25.6 \\
(29.6-21.5)\end{array}$ & $\begin{array}{c}25.3 \\
(29.3-21.3)\end{array}$ & $6 \mathrm{~m}-10 \mathrm{y}$ & $5 \mathrm{~m}-10 \mathrm{y}$ & Yes \\
\hline
\end{tabular}

$\begin{array}{ccccccccc}\text { Xie } 2008^{43} & \begin{array}{c}\text { Chinese } \\ \text { criteria } \\ \text { without } \\ \text { source }\end{array} & 23 / 7 & 25 / 5 & 20.70 \pm 4.35 & 22.20 \pm 4.27 & 1.70 \mathrm{y} \pm 2.46 \text { y } & 2.30 \mathrm{y} \pm 2.59 \mathrm{y} & \text { Yes } \\ & & & & & & \\ & & & & & \end{array}$

\begin{tabular}{|c|c|c|c|c|c|c|c|c|}
\hline $\mathrm{Xu}^{44} 2009$ & $\begin{array}{l}\text { Chinese } \\
\text { criteria }^{54}\end{array}$ & $37 / 25$ & $17 / 13$ & 21.3 & 21.5 & $1.72 \mathrm{y}$ & $1.67 \mathrm{y}$ & Yes \\
\hline $\mathrm{Xu}^{45} 2010$ & $\begin{array}{l}\text { Chinese } \\
\text { criteria }\end{array}$ & $5 / 27$ & $6 / 26$ & 22.97 & 22.63 & & & Yes \\
\hline $\operatorname{Yan}^{46} 2006$ & $\begin{array}{l}\text { Chinese } \\
\text { criteria }^{53}\end{array}$ & $5 / 27$ & $6 / 26$ & $22.97(17-43)$ & $22.63(18-38)$ & $2 \mathrm{~m}-13 \mathrm{y}$ & $2 \mathrm{w}-13 \mathrm{y}$ & Yes \\
\hline Yang $^{47} 2008$ & NR & $31^{*}$ & $31 *$ & 2 & 2 & & & NR \\
\hline Yang $^{48} 2008$ & NR & $16 / 5$ & & 16 & -35 & & & Yes \\
\hline Zhang $^{49} 2007$ & $\begin{array}{l}\text { Chinese } \\
\text { criteria }^{54}\end{array}$ & $29 / 27$ & $28 / 28$ & $22.4(16-34)$ & $22.1(15-35)$ & $\begin{array}{c}2.2 \mathrm{y} \\
(15 \mathrm{~d}-10 \mathrm{y})\end{array}$ & $\begin{array}{c}2.3 \mathrm{y} \\
(16 \mathrm{~d}-11 \mathrm{y})\end{array}$ & Yes \\
\hline
\end{tabular}


Table 1. (Continued)

\begin{tabular}{|c|c|c|c|c|}
\hline Intervention & Control & $\begin{array}{l}\text { Duration of } \\
\text { treatment }(w)\end{array}$ & Outcome & Adverse events \\
\hline $\begin{array}{l}\text { Acupuncture at GB14, SI18, } \\
\text { LI4, LI11, ST44, SP6, } \\
\text { GB34, } 30 \text { min once every } 2 \\
\text { days; acupoint injection of } \\
\text { dan shen extract } 4 \mathrm{~mL} \text { each } \\
\text { at BL } 13 \& \text { BL } 18 \text { or BL } \\
20 \& \text { BL } 231 \times / \text { every } 2 \text { d }\end{array}$ & $\begin{array}{l}\text { Acupuncture at GB14, } \\
\text { SI18, LI4, LI11, ST44, } \\
\text { SP6, GB34, with } \\
\text { additional points } \\
\text { BL13\& BL18 or BL20 } \\
\text { \& BL23,30 min once } \\
\text { every } 2 \text { days. }\end{array}$ & 8 & $\begin{array}{l}\text { Clinical symptom; } \\
\text { response to } \\
\text { intervention; } \\
\text { recurrence rate; } \\
\text { quality of life; } \\
\text { adverse events }\end{array}$ & No \\
\hline $\begin{array}{l}\text { Minocycline capsule, } 100 \mathrm{mg} \text {, } \\
\text { daily at bedtime; catgut } \\
\text { embedding LI } 4, \mathrm{LI} 11, \mathrm{ST} \\
36, \mathrm{ST} 37, \mathrm{BL} 25 \& \mathrm{BL} 21 \\
1 \times / \mathrm{w} \text {; tretinoin cream, } \\
0.025 \% \text {, applied to lesions } \\
\text { daily at bedtime; } \\
\text { clindamycin gel, } 0.1 \% \text {, } \\
\text { applied to lesions } 1 \times / \mathrm{d} \text { in } \\
\text { daytime }\end{array}$ & $\begin{array}{l}\text { Minocycline capsule, } \\
100 \mathrm{mg} \text {, daily at } \\
\text { bedtime; tretinoin } \\
\text { cream, } 0.025 \% \text {, applied } \\
\text { to lesions daily at } \\
\text { bedtime; clindamycin } \\
\text { gel, } 0.1 \% \text {, applied to } \\
\text { lesions } 1 \times / \mathrm{d} \text { in } \\
\text { daytime }\end{array}$ & 6 & $\begin{array}{l}\text { Skin lesion count; } \\
\text { Response to } \\
\text { intervention; } \\
\text { adverse effect; } \\
\text { recurrence rate; } \\
\text { IgG test }\end{array}$ & $\begin{array}{l}\text { Yes ( } 3 \text { cases in } \\
\text { intervention group \& } 6 \\
\text { cases in control group } \\
\text { reported dizziness; } 2 \\
\text { cases in control } \\
\text { reported mild stomach } \\
\text { ache) }\end{array}$ \\
\hline $\begin{array}{l}\text { Herbal decoction, } 150 \mathrm{~mL}, \\
\text { twice daily; acupuncture } \\
\text { around the lesion area } \\
30 \text { min once every } 2 \text { days }\end{array}$ & $\begin{array}{l}\text { Herbal decoction, } 150 \mathrm{~mL} \text {, } \\
\text { twice daily }\end{array}$ & 4 & $\begin{array}{l}\text { Response to } \\
\text { intervention }\end{array}$ & NR \\
\hline $\begin{array}{l}\text { Acupuncture on skin lesion } \\
\text { area } 20 \mathrm{~min} \text {, three times } \\
\text { weekly; Pi Pa Qing Fei } \\
\text { herbal decoction, } 50 \mathrm{~mL} \text {, } \\
2 \times / \mathrm{w}\end{array}$ & $\begin{array}{l}\text { Pi Pa Qing Fei herbal } \\
\text { decoction, } 50 \mathrm{~mL} \text {, twice } \\
\text { daily }\end{array}$ & 4 & $\begin{array}{l}\text { Response to } \\
\text { intervention; } \\
\text { WHO QOL- } \\
\text { BREF; lesion } \\
\text { count }\end{array}$ & No \\
\hline $\begin{array}{l}\text { Facial acupuncture on lesion } \\
\text { area, body acupuncture at } \\
\text { LI } 11 \text {, SP } 6 \text {, LR } 3 \& \text { KI3, } \\
20 \text { min, } 3 \times / \mathrm{w}, 10 \text { sessions } \\
\text { as } 1 \text { course; herbal } \\
\text { decoction, } 50 \mathrm{~mL}, 2 \times / \mathrm{d} \text {; } \\
\text { topical San Huang lotion } \\
\text { applied to lesions }\end{array}$ & $\begin{array}{l}\text { Herbal decoction, } 50 \mathrm{~mL} \text {, } \\
2 \times / \mathrm{d} \text {; topical San } \\
\text { Huang lotion applied to } \\
\text { lesions }\end{array}$ & 4 & $\begin{array}{l}\text { Response to } \\
\text { intervention }\end{array}$ & NR \\
\hline $\begin{array}{l}\text { Compound Betamethasone } \\
\text { injection, } 1 \mathrm{~mL} \text { with } 2 \% \\
\text { lidocaine } 1-5 \mathrm{~mL} \text { injection, } \\
\text { at } 2 \text { of } 4 \text { acupoints: ST } 8 \text {, } \\
\text { EX-HN 5, SJ 17, ST 6, ST } \\
2, \mathrm{ST} 4, \mathrm{CV} 24 \text {; viaminate } \\
25-50 \mathrm{mg}, 3 \times / \mathrm{d} \text {; } \\
\text { roxithromycin } 150 \mathrm{mg} \\
2 \times / \mathrm{d}\end{array}$ & $\begin{array}{l}\text { Viaminate, } 25-50 \mathrm{mg}, \\
3 \times / \mathrm{d} ; \text { roxithromycin, } \\
150 \mathrm{mg}, 2 \times / \mathrm{d}\end{array}$ & 8 & $\begin{array}{l}\text { Response to } \\
\text { intervention }\end{array}$ & Yes \\
\hline $\begin{array}{l}\text { Autologous blood injection, } \\
1 \mathrm{~mL} \text {, at LI } 11 \& \text { ST } 36 \\
1 \times \text { /every } 3 \mathrm{~d}\end{array}$ & $\begin{array}{l}\text { Erythromycin tablets, } \\
0.5 \mathrm{~g}, 3 \times / \mathrm{d} \text {; } \\
\text { ketoconazole cream } \\
\text { applied to lesions } 1 \times / \mathrm{d}\end{array}$ & 4 & $\begin{array}{l}\text { Response to } \\
\text { intervention }\end{array}$ & NR \\
\hline $\begin{array}{l}\text { Pi Pa Qing Fei decoction } \\
\text { modified according to } \\
\text { syndrome differentiation, } \\
\text { twice daily; dan shen }\end{array}$ & $\begin{array}{l}\text { Pi Pa Qing Fei decoction } \\
\text { modified according to } \\
\text { syndrome } \\
\text { differentiation, } 2 \times / \mathrm{d}\end{array}$ & 6 & $\begin{array}{l}\text { Response to } \\
\text { intervention }\end{array}$ & NR \\
\hline
\end{tabular}


TABle 1. (Continued)

\begin{tabular}{|c|c|c|c|c|c|c|c|c|}
\hline \multirow{2}{*}{$\begin{array}{l}\text { Study 1st } \\
\text { author \& ref. }\end{array}$} & \multirow{2}{*}{$\begin{array}{c}\text { Diagnostic } \\
\text { criteria }\end{array}$} & \multicolumn{2}{|c|}{ Sample size $(M / F)$} & \multicolumn{2}{|c|}{ Sample age (range, $y$ ) } & \multicolumn{2}{|c|}{ Disease duration (average) } & \multirow{2}{*}{$\begin{array}{c}\text { Baseline } \\
\text { data } \\
\text { comparable? }\end{array}$} \\
\hline & & $I$ & $C$ & $I$ & $C$ & $I$ & $C$ & \\
\hline Zhang $^{50} 2008$ & NR & $25 / 18$ & $28 / 15$ & $15-\sim 38$ & $13-\sim 35$ & $1 \mathrm{w}-8 \mathrm{y}$ & $1 \mathrm{w}-6 \mathrm{y}$ & NR \\
\hline
\end{tabular}

\begin{tabular}{|c|c|c|c|c|c|c|c|}
\hline Zhang $^{51} 2006$ & $\begin{array}{c}\text { Nonstandard } \\
\text { Chinese } \\
\text { criteria }\end{array}$ & $27 / 51$ & $30 / 52$ & 24.2 & 22.7 & $1 \mathrm{y}-10 \mathrm{y}$ & $6 \mathrm{~m}-\sim 7 \mathrm{y}$ \\
\hline Zhang $^{52} 2010$ & $\begin{array}{l}\text { Chinese } \\
\text { criteria } \\
\text { without } \\
\text { source }\end{array}$ & $57 / 55$ & $51 / 47$ & $22.5 \pm 4.3$ & $21.3 \pm 4.5$ & $3.3 \mathrm{~m} \pm 2.1 \mathrm{~m}$ & $3.6 \mathrm{~m} \pm 2.3 \mathrm{~m}$ \\
\hline
\end{tabular}

compared acupoint stimulation therapies and pharmaceutical medication; thus, blinding could not be applied for patients and researchers. Six trials ${ }^{13,25,26,32,45,46}$ reported the number of dropouts, but none used intention-to-treat (ITT) analysis. None of the trials mentioned sources of financial support.

\section{Effect Estimates}

Because of variations in study quality, participant characteristics, intervention types, controls, and outcome measures, results of most trials could not be synthesized by quantitative methods. Therefore, qualitative methods were used (Table 2).

Therapeutic effect of acupuncture. Therapeutic effect of acupuncture for acne was evaluated in 22 studies. Five studies $^{13-16,32}$ compared acupuncture with pharmaceutical medications. Nine trials 12,19,24,25,27,28,44-46 compared acupuncture plus herbal medicine with herbal medicine alone. Two trials ${ }^{21,33}$ compared acupuncture plus acupoint injection with acupoint injection alone. Two trials ${ }^{23,41}$ compared acupuncture plus a herbal facial mask with a facial mask alone. One trial ${ }^{18}$ compared acupuncture plus cupping therapy with cupping therapy alone. One trial ${ }^{35}$ compared acupuncture plus guasha (scraping) with guasha alone. One trial ${ }^{26}$ compared acupuncture plus herbal medicine and acupoint injection with herbal medicine and acupoint injection alone.

Nineteen trials ${ }^{12-16,18,19,21,23-25,27,28,32,33,41,44-46}$ were included in four meta-analyses. There were a significant dif- ference in the number of cured patients between acupuncture plus herbal medicine and herbal medicine alone (RR: $1.60 ; 95 \%$ CI: $1.19-2.14 ; P=0.002$; random model; $I^{2}=46 \% ; 9$ trials), and between acupuncture plus herbal facial mask and herbal facial mask alone $(R R: 2.14$; 95\% CI: $1.29-3.55 ; P=0.003$; fixed model; $I^{2}=0 \% ; 2$ trials). No difference was seen in the comparison between acupuncture and pharmaceutical medications ( $R R: 1.49$; 95\% CI: 0.82-2.73; $P=0.19$; random model; $I^{2}=54 \%$; 5 trials), and in the comparison between acupuncture plus acupoint injection and acupoint injection alone ( $R R: 2.00$; 95\% CI: 0.64-6.29, $P=0.24$; fixed model; $I^{2}=0 \% ; 2$ trials).

Three trials ${ }^{13,19,45}$ reported changes in skin-lesion count. One study $^{13}$ that used a skin-lesion scoring system, ${ }^{53}$ showed that acupuncture was superior to pharmaceutical medication for reducing the skin-lesion area (MD: -26.95 ; 95\% CI: -31.84 to $-22.06 ; P<0.00001 ; 1$ trial). The remaining two trials compared acupuncture plus herbal medicine with herbal medicine alone. One of the trials ${ }^{45}$ found that a combination of acupuncture and herbal medicine was better than herbal medicine alone for reducing skin lesions (MD: -13.88 ; 95\% CI: -19.17 to -8.59 ; $P<0.00001,1$ trial), while the other trial ${ }^{19}$ showed no difference between the comparison treatments (MD: -0.97 ; 95\% CI: -3.06 to $1.12 ; P=0.36 ; 1$ trial).

QoL (Acne-QoL) ${ }^{57}$ was assessed in one trial, ${ }^{14}$ finding that, compared with pharmaceutical medication, acupuncture appeared to significantly improve self-perception (MD: $3.40 ; 95 \%$ CI: $2.16-4.64 ; P<0.00001 ; 1$ trial), social 
TABle 1. (Continued)

\begin{tabular}{|c|c|c|c|c|}
\hline Intervention & Control & $\begin{array}{l}\text { Duration of } \\
\text { treatment }(w)\end{array}$ & Outcome & Adverse events \\
\hline $\begin{array}{l}\text { Bloodletting, followed by } \\
\text { flash cupping \& } 15 \text { min of } \\
\text { cup retention at at tender } \\
\text { Transporting points, at } \mathrm{GV} \\
14 \& \text { at additional points } \\
\text { according to syndrome } \\
\text { differentiation; treat } \\
1 \times / \text { every } 2 \mathrm{~d}\end{array}$ & $\begin{array}{l}\text { Tetracycline tablets } \\
500 \mathrm{mg} 1 \times / \mathrm{d} \text {; Cuo } \\
\text { Chuang Ping ointment } \\
\text { applied to lesions } 2 \times / \mathrm{d}\end{array}$ & 4 & $\begin{array}{l}\text { Response to } \\
\text { intervention }\end{array}$ & No \\
\hline $\begin{array}{l}\text { Acupuncture \& moxibustion, } \\
1 \times / \text { every other } \mathrm{d} ; 5 \\
\text { sessions as } 1 \text { course; } 3 \\
\text { courses total }\end{array}$ & $\begin{array}{l}\text { Acupuncture } 1 \times / \text { every } \\
\text { other d; } 5 \text { sessions as } 1 \\
\text { course; } 3 \text { courses total }\end{array}$ & 9 & $\begin{array}{l}\text { Response to } \\
\text { intervention }\end{array}$ & NR \\
\hline $\begin{array}{l}\text { Acupoint catgut embedding } \\
\text { at BL 13, BL 15, BL 18, } \\
\text { BL 16, BL 20, BL 21, ST } \\
\text { 36, LI 11, \& SP10; } \\
\text { additional points selected } \\
\text { according to syndrome } \\
\text { differentiation; treat } \\
1 \times / \text { every } 2 \mathrm{w}\end{array}$ & $\begin{array}{l}\text { Viaminate capsules, } \\
50 \mathrm{mg}, 2 \times / \mathrm{d} \text {; fusidic } \\
\text { acid cream applied to } \\
\text { lesions } 2 \times / \mathrm{d}\end{array}$ & 12 & $\begin{array}{l}\text { Response to } \\
\text { intervention }\end{array}$ & $\begin{array}{l}\text { Yes ( } 5 \text { cases in } \\
\text { intervention group \& } 4 \\
\text { cases in control } \\
\text { reported dry lips) }\end{array}$ \\
\hline
\end{tabular}

function (MD: 2.30; 95\% CI: 1.23-3.37; $P<0.0001 ; 1$ trial), and emotional function (MD 2.30; 95\% CI: 0.74-3.86; $P=0.004 ; 1$ trial).

Therapeutic effect of cupping therapy. Ten trials evaluated the effectiveness of cupping therapy for acne. Of the ten trials, four ${ }^{34,38,40,50}$ compared cupping therapy with pharmaceutical medications, three trials ${ }^{11,20,31}$ compared cupping therapy plus herbal medicine with herbal medicine alone, two trials ${ }^{30,37}$ compared cupping plus acupuncture with acupuncture alone, and one trial ${ }^{17}$ compared cupping plus a herbal facial mask with herbal facial mask alone.

Meta-analysis showed that cupping therapy was significantly better than pharmaceutical medications, such as tanshinone, tetracycline, and ketokonazole (RR: $2.11 ; 95 \%$ CI: 1.45-3.07; $P<0.0001$; fixed model; $I^{2}=6 \%, 4$ trials). Furthermore, cupping therapy combined with herbal medicine (RR: $1.91 ; 95 \%$ CI: $1.32-2.74 ; P=0.0005$; fixed model; $I^{2}=0 \% ; 3$ trials) or acupuncture $(R R: 1.79 ; 95 \%$ CI: $1.12-$ $2.86 ; P=0.01$; fixed model; $I^{2}=6 \% ; 2$ trials) was superior to herbal medicine or acupuncture alone. However, no difference was found between cupping plus a facial mask and a facial mask alone (RR: $1.58 ; 95 \%$ CI: $0.72-3.45 ; P=0.25 ; 1$ trial). As each comparison had fewer than five trials, it was not meaningful to conduct a funnel-plot analysis.
Therapeutic effect of acupoint injection. Seven trials evaluated the effect of acupoint injection for acne. Of the seven trials, three $22,39,48$ compared acupoint injection with pharmaceutical medication, and four trials compared acupoint injection plus other treatment with other treatment alone (pharmaceutical medication, ${ }^{29,47}$ acupuncture, ${ }^{42}$ and herbal medicine ${ }^{49}$ ).

Meta-analysis showed that acupoint injection used alone (RR: $1.51 ; 95 \%$ CI: 1.13-2.03; $P=0.006$; fixed model; $I^{2}=0 \% ; 3$ trials) and combined with pharmaceutical medication $(R R: 1.49 ; 95 \%$ CI: $1.12-1.99 ; P=0.007$; fixed model, $I^{2}=0 \% ; 2$ trials) were significantly better than

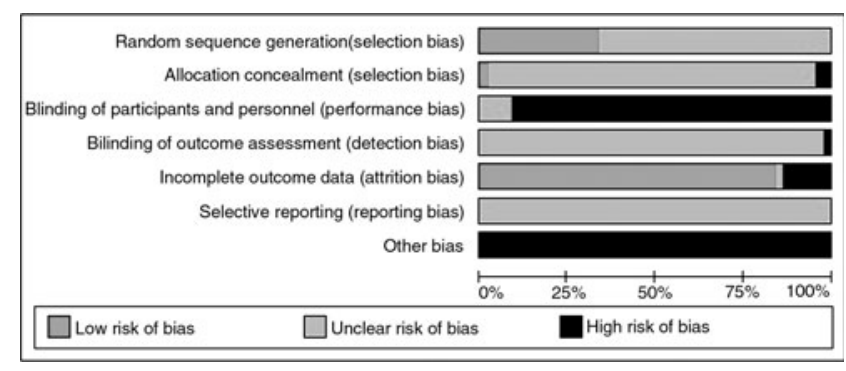

FIG. 2. Risk of bias graph shows the review authors' judgments about each risk of bias item, presented as percentages across all included studies. 
Table 2. Effect of Estimates of Acupoint Stimulation Treatment for Acne on Increasing Numbers of Cured Patients in 43 Randomized Controlled Trials

\begin{tabular}{lcc}
\hline $\begin{array}{l}\text { Study } 1 \text { st } \\
\text { author and ref. }\end{array}$ & Effect estimates \\
\hline
\end{tabular}

\section{Acupuncture}

1.1 Acupuncture versus pharmaceutical medications

Fan $2010^{13} \quad$ Acupuncture with fire needling versus topical tazarotene cream \& clindamycin gel

Gong $2005^{14} \quad$ Body acupuncture + ear acupressure versus licorsinc capsule

Han $2010^{15}$

He $2009^{16}$

Abdominal acupuncture versus isotretinoin capsule

Abdominal \& facial acupuncture versus topical metronidazole solution

Mao $2008^{32}$

Acupuncture versus minocycline

Overall (random, $I^{2}=54 \%$ )

Overall (fixed, $I^{2}=54 \%$ )

1.2 Acupuncture +other treatments versus other treatments alone

1.2.1 Acupuncture + herbal medicine versus herbal medicine alone

Cheng 2010 $\quad$ Ear acupressure + Bai Xian Xia Ta Re tablet versus Bai Xian Xia Ta Re tablet alone

Huang $2011^{19}$

Acupuncture + Pi Pa Qing Fei decoction \& herbal facial mask versus herbal decoction \& facial mask alone

Lan $2004^{24}$

Acupuncture + Qin Shen Fen Ci Qing oral liquid versus Qin Shen Fen $\mathrm{Ci}$ Qing liquid alone

Li $1995^{25} \quad$ Auricular therapy + herbal decoction versus herbal decoction alone

Li $2011^{27}$

Li $2011^{28}$

$\mathrm{Xu} 2009^{44}$

Xu $2010^{45}$

Yan $2006^{46}$

Acupuncture + herbal decoction and She Dan cream external application versus herbal decoction \& topical She Dan cream alone

Acupuncture + Yang He decoction versus Yang He decoction alone Acupuncture + herbal decoction versus herbal decoction alone

Acupuncture + Pi Pa Qing Fei decoction versus Pi Pa Qing Fei decoction alone

Body \& facial acupuncture + herbal decoction \& topical San Huang lotion versus herbal decoction \& topical San Huang lotion alone

Overall (random, $I^{2}=46 \%$ )

Overall (fixed, $I^{2}=46 \%$ )

1.2.2 Acupuncture + acupoint injection versus acupoint injection alone

Huang $2009^{21} \quad$ Electroacupuncture plus autologous blood injection versus autologous blood injection alone

Mi 2011 Acupuncture plus acupoint injection versus acupoint injection alone

Overall (random, $I^{2}=0 \%$ )

Overall (fixed, $I^{2}=0 \%$ )

1.2.3 Acupuncture + cupping therapy versus cupping therapy alone

Hou $2009^{18} \quad$ Auricular therapy + wet cupping versus wet cupping alone

1.2.4 Acupuncture + herbal facial mask versus herbal facial mask alone

Jin $2009^{23} \quad$ Body \& facial acupuncture + herbal facial mask versus herbal facial mask alone

Xie $2009^{41} \quad$ Acupuncture + herbal facial mask versus herbal facial mask alone

Overall (random, $I^{2}=0 \%$ )

Overall (fixed, $I^{2}=0 \%$ )

1.2.5 Acupuncture plus guasha (scraping) versus guasha alone

Wang $2011^{35} \quad$ Acupuncture + guasha versus guasha alone
$2.43[0.74,7.99]$

$2.00[0.20,20.33]$

$0.80[0.50,1.27]$

$1.38[0.45,4.24]$

$2.34[1.23,4.47]$

$R R 1.49$ [0.82, 2.73]

$R R$ 1.36 [0.97, 1.89]

0.19

0.07

$1.33[1.02,1.74]$

$2.00[0.78,5.15]$

$1.49[0.75,2.96]$

$2.86[1.17,6.97]$

$1.07[0.79,1.44]$

$5.10[1.72,15.11]$

$1.37[0.60,3.12]$

$2.00[0.67,5.98]$

$2.00[0.67,5.98]$

$R R$ 1,60 [1.19, 2.14]

$R R$ 1.56 [1.30, 1.89]

0.002

$<0.00001$

$2.00[0.40,10.11]$

$2.00[0.40,10.11]$

$R R 2.00[0.64,6.29]$

$R R 2.00[0.64,6.29]$

0.24

0.24

$1.47[1.00,2.18]$

0.05

$2.17[0.64,7.35]$

2.13 [1.24, 3.68]

$R R$ 2.14 [1.30, 3.52]

$R R$ 2.14 [1.29, 3.55]

0.003

0.003

$3.00[0.92,9.74]$

0.07 
TABle 2. (Continued)

\begin{tabular}{|c|c|}
\hline $\begin{array}{l}\text { Study } 1 \text { st } \\
\text { author and ref. }\end{array}$ & Comparisons \\
\hline \multicolumn{2}{|c|}{ 1.2.6 Acupuncture plus herbal medicine and acupoint injection versus herbal medic } \\
\hline Li $2009^{26}$ & $\begin{array}{l}\text { Auricular therapy }+ \text { herbal decoction, topical medicine, acupoin } \\
\text { injection versus herbal decoction, topical medicine, \& acupo } \\
\text { injection }\end{array}$ \\
\hline \multicolumn{2}{|c|}{ 2. Cupping therapy } \\
\hline \multicolumn{2}{|c|}{ 2.1 Cupping + other interventions versus other interventions alone } \\
\hline \multicolumn{2}{|c|}{ 2.1.1 Cupping + herbal medicine versus herbal medicine alone } \\
\hline Chen $2009^{11}$ & Wet cupping + herbal decoction versus herbal decoction alone \\
\hline Huang $2010^{19}$ & $\begin{array}{l}\text { Wet cupping + herbal preparation, topical cream versus herbal } \\
\text { preparation \& topical cream }\end{array}$ \\
\hline Lu $2011^{31}$ & Wet cupping + herbal decoction versus herbal decoction alone \\
\hline
\end{tabular}

Overall (random, $I^{2}=0 \%$ )

Overall (fixed, $I^{2}=0 \%$ )

2.1.2 wet cupping plus acupuncture versus acupuncture alone

Liu $2009^{30} \quad$ Flash cupping plus acupuncture versus acupuncture alone

Wang $2007^{37} \quad$ Moving and wet cupping + acupuncture versus acupuncture alone

Overall (random, $I^{2}=0 \%$ )

Overall (fixed, $I^{2}=0 \%$ )

2.1.3 wet cupping plus facial mask versus facial mask

Hong $2011^{17}$ Wet cupping + herbal facial mask versus herbal facial mask alone

2.2 wet cupping versus pharmaceutical medication

Song $2011^{34} \quad$ Wet cupping versus vitamin B/metronidazole/erythromycin, metronidazole cream/benzoyl peroxide gel/sulfur/Mei Lu Xiao Cuо cream

Wu $2010^{38}$

Wu $2008^{40}$

Zhang $2008^{50}$

Wet cupping versus tonshinone

Wet cupping versus tetracycline \& ketoconazole cream

Wet cupping versus tetracycline

Overall (random, $I^{2}=6 \%$ )

Overall (fixed, $I^{2}=6 \%$ )

3. Acupoint injection

3.1 Acupoint injection versus pharmaceutical medication

Huang $2011^{22} \quad$ Autologous blood acupoint injection versus viaminate capsule \& tetracycline tablet

Wu $1998^{39} \quad$ Yu xing cao \& dang gui acupoint injection versus metronidazole tablet \& minocycline tablet

Yang $2008^{48}$

Autologous blood acupoint injection versus erythromycin tablet \& topical ketoconazole

Overall (random, $\mathrm{I}^{2}=\mathbf{0} \%$ )

Overall (fixed, $\mathrm{I}^{2}=0 \%$ )

3.2 Acupoint injection +other treatment versus other treatment alone

3.2.1 Acupoint injection + pharmaceutical medication versus pharmaceutical medication alone Liu 2008 $29 \quad$ Autologous blood acupoint injection + vitamin B/zinc gluconate tablet/Luo Hong enzyme capsule/Pi Pa Qing Fei decoction/acne tincture $\&$ acupoint injection versus pharmaceutical medication $\&$ acupoint injection

Yang $2008^{47} \quad$ Compound betamethasone injection with $2 \%$ lidocaine acupoint injection + viaminate \& roxithromycin versus viaminate \&

Overall (random, $I^{2}=0 \%$ ) roxithromycin

Overall (fixed, $I^{2}=0 \%$ )
Effect estimates

[95\% CI]

$P$

0.50

$1.38[0.55,3.49]$

$2.33[0.67,8.18]$

$2.06[1.33,3.18]$

1.44 [0.67, 3.12]

$R R 1.92$ [1.34, 2.76]

$R R$ 1.91 [1.32, 2.74]

0.0004

0.0005

$1.91[0.99,3.72]$

1.67 [0.87, 3.20]

$R R$ 1,79 [1.12, 2.86]

$R R 1.79$ [1.12, 2.86]

0.01

0.01

$1.58[0.72,3.45]$

0.25

$2.00[0.80,4.98]$

$1.07[0.45,2.56]$

$2.50[1.31,4.77]$

$2.75[1.38,5.48]$

$R R 2.10$ [1.42, 3.11]

$R R 2.11$ [1.45, 3.07]

0.0002

$<0.0001$

2.13 [1.09, 4.16]

$1.67[1.09,2.56]$

$1.29[0.71,2.36]$

$R R$ 1.51 [1.13, 2.03]

$R R$ 1.64 [1.20, 2.24]

0.006

0.002

$1.43[1.03,1.98]$

$1.78[0.93,3.40]$

$R R$ 1.49 [1.12, 1.99]

$R R 1.79$ [1.12, 2.86]
0.007

0.01 
TABle 2. (CONTINUED)

\begin{tabular}{|c|c|c|c|}
\hline $\begin{array}{l}\text { Study } 1 \text { st } \\
\text { author and ref. }\end{array}$ & Comparisons & $\begin{array}{l}\text { Effect estimates } \\
{[95 \% \mathrm{CI}]}\end{array}$ & $P$ \\
\hline \multicolumn{4}{|c|}{ 3.2.2 Acupoint injection + acupuncture versus acupuncture alone } \\
\hline Xie $2011^{42}$ & $\begin{array}{l}\text { Acupoint injection with danshen extract + acupuncture versus } \\
\text { acupuncture alone }\end{array}$ & $1.45[0.46,4.59]$ & 0.53 \\
\hline \multicolumn{4}{|c|}{ 3.2.3 Acupoint injection + herbal medicine versus herbal medicine alone } \\
\hline Zhang $2007^{49}$ & $\begin{array}{l}\text { Acupoint injection with danshen extract }+P i \text { Pa Qing Fei decoction } \\
\text { versus herbal decoction }\end{array}$ & $1.08[0.83,1.41]$ & 0.55 \\
\hline \multicolumn{4}{|c|}{ 4. Acupoint catgut embedding } \\
\hline \multicolumn{4}{|c|}{ 4.1 Acupoint catgut embedding versus Western medication } \\
\hline Zhang $2010^{52}$ & $\begin{array}{l}\text { Acupoint catgut embedding versus viaminate capsules \& fusidic } \\
\text { acid cream }\end{array}$ & $1.57[1.15,2.15]$ & 0.004 \\
\hline \multicolumn{4}{|c|}{ 4.2 Acupoint catgut embedding +other treatment versus other treatment alone } \\
\hline \multicolumn{4}{|c|}{ 4.2.1 Acupoint catgut embedding + pharmaceutical medication versus pharmaceutical medication alone } \\
\hline Xie $2008^{43}$ & $\begin{array}{l}\text { Acupoint catgut embedding + minocycline capsule, tretinoin cream/ } \\
\text { clindamycin gel versus minocycline capsule, tretinoin cream/ } \\
\text { clindamycin gel }\end{array}$ & $1.40[0.50,3.92]$ & 0.52 \\
\hline \multicolumn{4}{|c|}{ 4.2.2 Acupoint catgut embedding + herbal medicine versus herbal medicine alone } \\
\hline Wang $2009^{36}$ & $\begin{array}{l}\text { Acupoint catgut embedding }+ \text { herbal decoction versus herbal } \\
\text { decoction alone }\end{array}$ & $1.85[1.24,2.77]$ & 0.003 \\
\hline \multicolumn{4}{|l|}{ 5. Moxibustion } \\
\hline \multicolumn{4}{|c|}{ 5.1 Moxibustion + acupuncture versus acupuncture alone } \\
\hline Chen $2007^{10}$ & Moxibustion + acupuncture versus acupuncture alone & $1.67[0.33,8.48]$ & \\
\hline Zhang $2006^{51}$ & Moxibustion + acupuncture versus acupuncture alone & $1.46[1.04,2.05]$ & \\
\hline \multicolumn{2}{|c|}{ Overall (random, $I^{2}=0 \%$ ) } & $R R 1.47[1.05,2.05]$ & 0.03 \\
\hline \multicolumn{2}{|c|}{ Overall (fixed, $I^{2}=0 \%$ ) } & $R R 1.47[105,2.07]$ & $\mathbf{0 . 0 3}$ \\
\hline
\end{tabular}

$\mathrm{CI}$, confidence interval; $R R$, risk ratio.

medication alone. However, no difference was found between acupoint injection combined with herbal medicine (RR: $1.08 ; 95 \%$ CI: $0.83-1.41 ; P=0.55 ; 1$ trial) or acupuncture (RR: $1.45 ; 95 \%$ CI: $0.46-4.59 ; P=0.53 ; 1$ trial) compared with herbal medicine or acupuncture alone. As each comparison had fewer than five trials, it was not meaningful to conduct a funnel-plot analysis.

One trial ${ }^{42}$ reported QoL scores and recurrence rate. Results showed no difference between acupoint injection plus acupuncture and acupuncture alone in improving QoL (MD: $-1.76 ; 95 \% \mathrm{CI}:-3.80$ to $0.28 ; P=0.09 ; 1$ trial) and in reducing recurrence rate $(R R: 0.22 ; 95 \% \mathrm{CI}: 0.03-1.60$; $P=0.13 ; 1$ trial $)$.

Therapeutic effect of acupoint catgut embedding. Three trials ${ }^{36,43,52}$ evaluated the therapeutic effect of acupoint catgut embedding for acne. One study ${ }^{52}$ showed acupoint catgut embedding was superior to pharmaceutical medication in increasing the number of cured patients $(R R$ : 1.57; 95\% CI: $1.15-2.15 ; P=0.004 ; 1$ trial) and in reducing recurrence rate $(R R: 0.22 ; 95 \% \mathrm{CI}: 0.08-0.62 ; P=0.004 ; 1$ trial). One trial $^{36}$ showed acupoint catgut embedding combined with herbal medicine was superior to herbal medicine alone in increasing the number of cured patients ( $R R: 1.85$; 95\% CI: $1.24-2.77 ; P=0.003 ; 1$ trial). One study ${ }^{43}$ showed no difference between acupoint catgut embedding plus pharmaceutical medication and pharmaceutical medication alone in increasing the number of cured patients (RR: 1.40; 95\% CI: $0.50-3.92 ; P=0.52 ; 1$ trial), reducing skin-lesion area (MD: $2.67 ; 95 \% \mathrm{CI}:-0.07$ to $5.41 ; P=0.06 ; 1$ trial), and reducing recurrence rate (RR: $0.48 ; 95 \%$ CI: $0.12-1.88$; $P=0.29 ; 1$ trial $)$.

Therapeutic effect of moxibustion. Meta-analysis of two trials ${ }^{10,51}$ showed that a combination of moxibustion and acupuncture was better than acupuncture alone for increasing the number of cured patients (RR: $1.47 ; 95 \% \mathrm{CI}$ : 1.05-2.07; $P=0.03$; fixed model; $I^{2}=0 \% ; 2$ trials).

Adverse events. Twenty-nine trials did not mention adverse events. Of the fourteen trials ${ }^{10,11,13,19,27,30,31,36,42,43,45,47,50,52}$ that did report adverse events, seven trials ${ }^{11,27,30,31,42,45,50}$ found no adverse events in both intervention and control groups. The remaining seven trials reported mild adverse events in the intervention and control groups (Table 1), such as thirst, dizziness, redness and swelling of the treated site, 


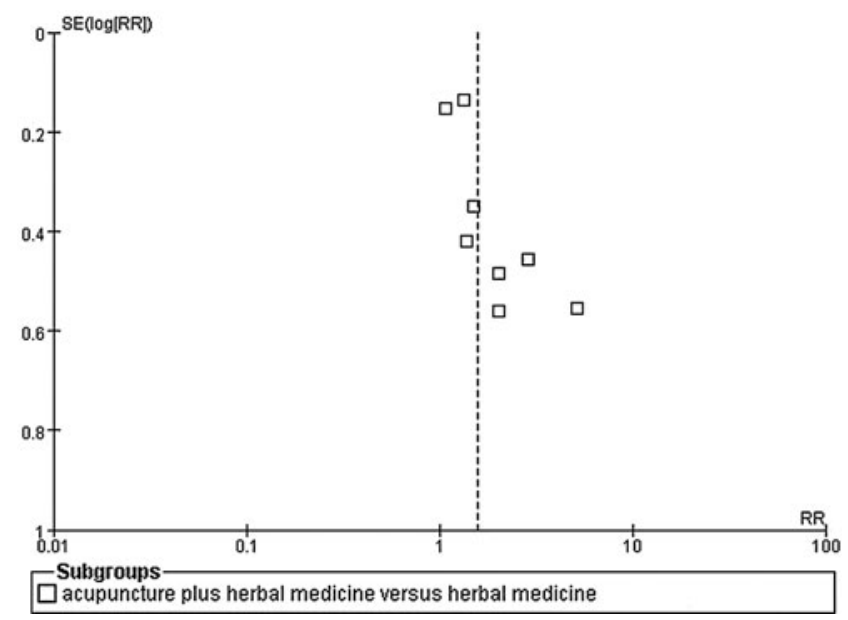

FIG. 3. Funnel plot of eight trials for the outcome of number of patients cured of acne. SE, standard error; $R R$, relative risk.

and pain or itching in the acupoint area. Serious adverse events were not reported.

Funnel-plot analysis. Funnel-plot analysis of eight trials showed significant asymmetry (Fig. 3).

\section{DISCUSSION}

This study's data demonstrated that, in the studies that were evaluated, acupuncture and cupping therapy used alone or in combination with pharmaceutical medication appeared to be more effective than pharmaceutical medication alone in increasing the number of cured patients with acne. However, further studies are needed to confirm this finding. The therapeutic effects of acupoint injection, acupoint catgut embedding, and moxibustion were unclear because there was insufficient evidence from the available studies.

Results of nine meta-analyses found that acupointstimulation therapies combined with other treatments were significantly more efficacious than other treatments applied alone. Cupping therapy and acupoint injection appeared to be superior to pharmaceutical medication, whereas no difference in efficacy was seen between acupuncture and pharmaceutical medication. Although across studies, the use of pharmaceutical medication was not guideline-based or dosage-consistent (Table 1), acupoint-stimulation therapies, including acupuncture, cupping, and acupoint injection may have an equivalent therapeutic effect as medication (antibiotics, antiprotozoal, licorsinc, isotretinoin) for acne. Given that no severe adverse events were reported in the included studies, the current authors believe that it would be worthwhile to conduct further, rigorously designed trials on acupoint-stimulation therapies for the treatment of acne.

This current review revealed that there remains a lack of well-designed studies on the treatment of acne using acu- point-stimulation therapies. Methodological quality of the studies included in this review was generally poor, indicating a high risk of bias. Inadequate application of randomization and absence of blinding were evident in the majority of trials, causing potential performance bias and detection bias, because patients and researchers were aware of the therapeutic interventions. Applying proper blinding methodology remains a challenge for studies on manualhealing therapies. Even so, at the very least, blinding of outcome assessors is highly recommended in such studies. ITT analysis was not applied in most of the trials and the funnel-plot indicated that these data may have publication bias. Intervention response using the ambiguous and subjective terminology of cure, markedly effective, effective, and ineffective was difficult to interpret and validate across studies. Consequently, any positive finding needs to be interpreted cautiously. Researchers of future studies should consider applying more robustly defined intervention response measurements, such as one of the existing acne grading scales. ${ }^{58}$ None of the trials reported sample-size calculation. The current authors strongly recommend that future RCTs include sample-size estimates to ensure adequate statistical power. Furthermore, sample-size calculation and analysis of outcomes should be based on the principle of ITT.

Twelve trials ${ }^{13,14,16,19,21,23,24,30,44-46,50}$ used skin-lesion ("ouch" point) areas as the main targets for stimulation, while other studies ${ }^{10-12,15,17,18,20,22,25-29,31-43,47-49,51,52}$ established acupoint prescriptions that were followed throughout the duration of the trials. The limited number of trials precluded the current authors from ascertaining the differences in therapeutic effects among these three types of acupoint-selection methods.

The potential asymmetry of the funnel-plot test (Fig. 3) of eight trials that examined acupuncture plus herbal medicine, compared with herbal medicine alone may have been caused by small study effects or even heterogeneity in intervention effects. Furthermore, as ongoing trials were not included, and, as all trials were conducted in China, there is a high potential for publication bias in the current review.

In summary, most of the existing trials were of small size and had a high risk of bias. Further high-quality, large-scale studies are needed to confirm the effectiveness of acupointstimulation therapy for treating acne. Randomization methods need to be described clearly and reported fully. Blinding of outcome assessors should be attempted as feasibly as possible to minimize performance and assessment biases. Outcome (response) measures utilizing acne-grading scales should be applied and should include contiguous data, such as skin-lesion scores from baseline to study completion. Analysis of outcomes based on the ITT principle is vital as is the application of sample-size calculation. Reporting of trials should adhere to the Consolidated Standards $O$ f Reporting Trials (CONSORT) ${ }^{59}$ to ensure clarity and completeness of reporting. 


\section{CONCLUSIONS}

Acupoint-stimulation therapy-especially when it is combined with other treatments - appears to be effective for treating acne. However, further large, rigorously designed trials are needed to confirm these findings.

\section{ACKNOWLEDGMENTS}

H.-j. Cao and J.-p. Liu were supported by the Research Capacity Establishment Grant (number 101207007) of Beijing University of Chinese Medicine. This work was also supported by the grant numbers 2009ZX09502-028 and 2011ZX09302-006-01-03(5). The authors thank Nissi $\mathrm{S}$. Wang MS, for content editing of this manuscript.

\section{DISCLOSURE STATEMENT}

No competing financial interests exist.

\section{REFERENCES}

1. Simonart T, Dramaix M. Treatment of acne with topical antibiotics: Lessons from clinical studies. Br J Dermatol. 2005; 153(2):395-403.

2. Ramos-e-Silva M, Carneiro SC. Acne vulgaris: Review and guidelines. Dermatol Nurs. 2009;21(2):63-68.

3. Oberemok SS, Shalita AR. Acne vulgaris, II: Treatment. Cutis. 2002;70(2):111-114.

4. Webster GF. Acne vulgaris. BMJ. 2002;325(7362):475-479.

5. Marqueling AL, Zane LT. Depression and suicidal behavior in acne patients treated with isotretinoin: A systematic review. Semin Cutan Med Surg. 2007;26(4):210-220.

6. Shen DH, Wu XF, Wang N. Acne and rosacea. Manual of Dermatology in Chinese Medicine. Seattle: Eastland Press; 1995:253-258.

7. Li B, Chai H, Du YH, Xiao L, Xiong J. Evaluation of therapeutic effect and safety for clinical randomised and controlled trials of treatment of acne with acupuncture and moxibustion [in Chinese]. Chinese Acupunct Moxibustion 2009;29(3):247-251.

8. Magin PJ, Adams J, Pond CD, Smith W. Topical and oral CAM in acne: A review of the empirical evidence and a consideration of its context. Complement Ther Med. 2006;14(1):62-76.

9. Higgins JPT, Green S, eds. Cochrane Handbook for Systematic Reviews of Interventions, Version 5.1.0 [updated March 2011]. The Cochrane Collaboration, 2011. Online document at: www.cochrane-handbook.org Accessed February 15, 2012.

10. Chen H, Fu YJ. Clinical Study on Warm Needling Method for Treating Acne Cysts' Nodules [in Chinese; Master's thesis]. Guangzhou, China: Guangzhou University of Chinese Medicine; 2007.

11. Chen YQ, Lai XS. Clinical Study on Bloodletting of BackShu Points for Treating Acne [in Chinese; Master's thesis]. Guangzhou, China: Guangzhou University of Chinese Medicine; 2009.
12. Cheng L. Clinical study of 50 cases of auricular acupressure with vaccaria seed for acne: Nursing guidance [in Chinese]. $J$ Qilu Nurs. 2010;16(11):39-40.

13. Fan Y, Huang Y. Clinical Research on Treatment of Acne Using Fireneedle Acupuncture [in Chinese; Master's thesis]. Chengdu, China: Chengdu University of Traditional Chinese Medicine; 2010.

14. Gong SB, Huang BY. Clinical Study on Treatment of Acne Vulgaris with Scattered Needling Combined with Auricular Point Adhesion [in Chinese; Master's thesis]. Fujian. China: Fujian University of Traditional Chinese Medicine; 2005.

15. Han B, Mi JP. Clinical Study on Acupuncture of Abdominal Acupoints for Treatment of Acne by Regulating the Liver and Kidney [in Chinese; Master's thesis]. Guangzhou, China: Guangzhou University of Chinese Medicine; 2010.

16. He XY. Clinical observation of acupuncture of abdominal and facial acupoints for treatment of 24 cases of facial acne [in Chinese]. Nei Mongol J Traditional Chinese Med. 2009; 28(8):33.

17. Hong TT, Wu LX. Cupping with bloodletting combined with Chinese medicinal facial mask for treatment of acne [in Chinese]. Shanghai J Acupunct Moxibustion. 2011;30(16): 387-388.

18. Hou GY, Cao L, Liu YP, Li HJ. Observation on the therapeutic effect of cupping with bloodletting combined with ear acupuncture on acne [in Chinese]. J Liaoning University Traditional Chinese Med. 2009;11(10):145-146.

19. Huang CQ, Huang Y. Clinical Observation on Treating Adolescent Acne Using Combination of Acupuncture with Oral and External Traditional Chinese Medicine [in Chinese; Master's thesis]. Guangzhou, China: Southern Medical University; 2011.

20. Huang J, Wei D, Wu JD. Cupping with bloodletting in the treatment of 76 cases of acne [in Chinese]. China Bio-Beauty. 2010;(1):19-21.

21. Huang RL, Li SH. Observations on the Therapeutic Effect of Acupoint Autologous Blood Injection Combined with Acupuncture of Ashi Points for the Treatment of Acne Vulgaris [in Chinese; Master's thesis]. Guangzhou, China: Guangzhou University of Chinese Medicine; 2009.

22. Huang Y, Wu YY, Ma WL, Pan HY, Jiang SM, Qu YB. Acupoint autologous blood injection combined with skin hygiene for treating acne [in Chinese]. Guangdong Med J. 2011;32(4):514-515.

23. Jin W. Acupuncture combined with external application of herbal medicine in the treatment of 58 patients with acne [in Chinese]. Acta Chinese Med Pharmacol. 2009;37(2):6869.

24. Lan D, Si TR, Zhao SL, Mao YL, Zhang HY. Clinical and experimental studies on acupuncture combined with medication for treatment of female delayed-type and persistent acne of different Chinese medicine syndrome types [in Chinese]. Zhong Guo Zhen Jiu 2004;24(6):379-382.

25. Li LF. Ear-point detection and observation on therapeutic effect of ear acupuncture in patients with acne [in Chinese]. Chinese Acupunct Moxibustion. 1995;(3):24-25.

26. Li M, Fan RQ. Investigation of the Treatment of Female Acne Vulgaris by Chinese Medicine Regulation of Liver-Kidney and Chong-Ren Channels [in Chinese; Master's thesis]. 
Guangzhou, China: Guangzhou University of Chinese Medicine; 2009.

27. Li Q, Sun YC. Acne Chinese medicine complex therapy effect observation analysis [in Chinese]. Chinese Manipulation Rehabil Med. 2011;38(1):175-177.

28. Li Y, He L, Liu BH, Zhang HR, Zhang P, Ai MM. Fire needle therapy combined with Yanghe decoction in the treatment of acne of yang deficiency type [in Chinese]. J Clin Acupunct Moxibustion. 2011;27(4):11-13.

29. Liu HL, Wang LX. Observation on therapeutic effect of acupoint autologous blood injection as an adjuvant treatment for acne vulgaris [in Chinese]. J Changzhi Med Coll. 2008; 22(6):452-453.

30. Liu HP, Liang B, He JB, Zhang CQ. Therapeutic effect of acupuncture combined with flash cupping for acne vulgaris [in Chinese]. Liaoning J Traditional Chinese Med. 2009; 36(8):1395-1397.

31. Lu LH, Zhu MH. Clinical Observations on Treating Acne (Chinese Medicine Type): Lung Wind Heat by Bloodletting and Cupping with Herbal Medicine on Dazhui Acupoint [in Chinese; Master's thesis]. Guangzhou, China: Guangzhou University of Chinese Medicine; 2011.

32. Mao L, Zhang MB. Clinical observations on acupuncture for the treatment of acne [in Chinese]. J Liaoning University Traditional Chinese Med. 2008;10(7):120-121.

33. Mi JP, Yu ZS. Clinical observations on therapeutic effect of acupuncture of abdominal acupoints in the treatment of cystic acne [in Chinese]. 2011 Annual Symposium of China Association of Acupuncture-Moxibustion, Beijing, China, August 19-22, 2011.

34. Song XW. Clinical observations on acupoint pricking therapy for treatment of acne [in Chinese]. Med JChinese People's Health. 2011;23(16):2008-2009.

35. Wang D, Fu YJ. Clinical Observations on Therapeutic Effect of Acupuncture and Guasha in the Treatment of Damp-Heat Type Acne [in Chinese; Master's thesis]. Guangzhou, China: Guangzhou University of Chinese Medicine; 2011.

36. Wang L, Feng ZH, Liu L. Acupoint catgut embedding combined with oral Chinese medicine in the treatment of 68 cases of acne [in Chinese]. Chinese J Dermatol Venereol Integr Traditional Western Med. 2009;8(3):168-169.

37. Wang QF, Wang GY. Observations on the efficacy of acupuncture and moxibustion plus bloodletting acupuncture and movable cupping in the treatment of acne [in Chinese]. Shanghai J Acupunct Moxibustion. 2007;26(12):20-21.

38. Wu FF, Yang SQ, Zhang SJ. Bloodletting acupuncture and cupping on Back-Shu acupoints in the treatment of adolescent acne [in Chinese]. J Qiqihar Medical Coll. 2010; 31(10): 1586.

39. Wu HX, Wang ZL. Clinical observations on therapeutic effect of 68 cases of acne treated with hydroacupuncture [in Chinese]. J Zhejiang Coll Traditional Chinese Med. 1998;22(4):40-41.

40. Wu YT. Therapeutic effect of bloodletting cupping therapy on the back in the treatment of acne [in Chinese]. J Practical Traditional Chinese Internal Med. 2008;22(10):61-32.

41. Xie CC, Zhong JN, Yang ZX. Clinical observations on therapeutic effect of acupuncture and medicinal facial mask in the treatment of acne [in Chinese]. Southern China J DermatoVenereol. 2009;16(3):193-195.
42. Xie GX, Ding H. Clinical Observations on Quality of Life After Treatment of Acne with Acupuncture and Injection [in Chinese; Master's thesis]. Guangzhou, China: Guangzhou University of Chinese Medicine; 2011.

43. Xie J, Wang WZ. Acupoint Catgut Embedding Combined with Minocycline in the Treatment of Acne due to Damp-Heat Syndrome [in Chinese; Master's thesis]. Hubei, China: Hubei University of Chinese Medicine; 2008.

44. Xu JP, Liu CZ. Observations on the therapeutic effect of oral Chinese medicine combined with acupuncture in the treatment of 62 cases of acne vulgaris [in Chinese]. Chinese $J$ Dermatol Venereol. 2009;23(1):55-56.

45. Xu RJ, Fu WB. Clinical Observations on Combination of Acupuncture with Herbs in the Treatment of Acne Vulgaris of Lung Wind-Heat Type [in Chinese; Master's thesis]. Guangzhou, China: Guangzhou University of Chinese Medicine; 2010.

46. Yan DY, Fan RQ. Clinical Observations on Quality of Life After Treatment of Acne with Traditional Chinese Herbs Combined with Facial Acupuncture [in Chinese; Master's thesis]. Guangzhou, China: Guangzhou University of Traditional Chinese Medicine; 2006.

47. Yang L. Compound betamethasone acupoint injection and vitamin A acid combination therapy in 31 cases of severe acne [in Chinese]. Nei Mongol J Traditional Chinese Med. 2008;27(11):95-96.

48. Yang QJ. Observations on acupoint autologous blood injection in 36 cases of acne [in Chinese]. China's Naturopathy. 2008;16(7):11.

49. Zhang F. Clinical observations on traditional Chinese medicine accompanied by hydro-acupuncture therapy in 56 cases of acne [in Chinese]. Liaoning J Traditional Chinese Med. 2007;34(10):1423-1424.

50. Zhang KX, Song SJ. Clinical observations on pricking with tri-ensiform needle and bloodletting cupping on back-shu points in the treatment of acne vulgaris [in Chinese]. World Health Dig. 2008;5(5):193-194.

51. Zhang L. Acupuncture and moxibustion treatment of 160 cases of acne [in Chinese]. Beijing J Traditional Chinese Med. 2006;25(8):497-498.

52. Zhang XP, Ran YY, Ma K. Treatment of acupoint catgut embedding with pharmaceutical medicine on 112 cases of acne [in Chinese]. Henan J Traditional Chinese Med. 2010; 30(5):504-505.

53. Zheng XY. Dermatology disease. In: Guiding Principle of Clinical Research on New Drugs of Traditional Chinese Medicine [in Chinese]. Beijing: China Medical Science Press; 2002:290-295.

54. State Administration of Traditional Chinese Medicine. ZY/ T001.8-94. Dermatology disease. In: Criteria of Diagnosis and Therapeutic Effect of Traditional Chinese Medicine [in Chinese]. Nanjing: Nanjing University Press; 1994:158.

55. Zhao B. Disease of skin appendages. In: Clinical Dermatology [in Chinese]. Jiangsu: Jiangsu Science and Technology Press; 2003:833-835.

56. Wang XS, Liao KH. Monograph No. 44: Disease of sebaceous glands and sweat glands. Yang Guoliang's Dermatology [in Chinese]. Shanghai: Shanghai Science and Technology Literature Press; 2005:725-726. 
CAO ET AL.

57. Fehnel SE, Mcleod LD, Brandman J, et al. Responsiveness of the acne-specific quality of life questionnaire (Acne-QoL) to treatment for acne vulgaris in placebo-controlled clinical trials. Qual Life Res. 2002;11(8):809-816.

58. Adityan B, Kumari R, Thappa DM. Scoring systems in acne vulgaris. Indian J Dermatol Venereol Leprol. 2009;75(3): 323-326.

59. The CONSORT Group. CONSORT Statement 2001Checklist: Items to Include When Reporting a Randomized Trial. Online document at: www.consort-statement.org. Accessed: March 3, 2012.
Address correspondence to: Jian-ping Liu, PhD Centre for Evidence-based Chinese Medicine Beijing University of Chinese Medicine 88\# Mail Box

Bei San Huan Dong Lu 11 Chaoyang District, Beijing 100029

China

E-mail: jianping_1@hotmail.com 